\title{
A two-sex model of human papillomavirus infection: Vaccination strategies and a case study
}

\author{
Shasha Gao ${ }^{\mathrm{a}}$, Maia Martcheva ${ }^{\mathrm{a}, *}$, Hongyu Miao $^{\mathrm{b}}$, Libin Rong $^{\mathrm{a}, *}$ \\ ${ }^{a}$ Department of Mathematics, University of Florida, Gainesville, FL 32611 \\ ${ }^{b}$ Department of Biostatistics and Data Science, University of Texas Health Science Center at Houston, TX 77030
}

\begin{abstract}
Vaccination is effective in preventing human papillomavirus (HPV) infection. It still remains debatable whether males should be included in a vaccination program and unclear how to allocate the vaccine in genders to achieve the maximum benefits. In this paper, we use a two-sex model to assess HPV vaccination strategies and use the data from Guangxi Province in China as a case study. Both mathematical analysis and numerical simulations show that the basic reproduction number, an important indicator of the transmission potential of the infection, achieves its minimum when the priority of vaccination is given to the gender with a smaller recruit rate. Given a fixed amount of vaccine, splitting the vaccine evenly usually leads to a larger basic reproduction number and a higher prevalence of infection. Vaccination becomes less effective in reducing the infection once the vaccine amount exceeds the smaller recruit rate of the two genders. In the case study, we estimate the basic reproduction number is 1.0333 for HPV 16/18 in people aged 15-55. The minimal bivalent HPV vaccine needed for the disease prevalence to be below $0.05 \%$ is 24050 per year, which should be given to females. However, with this vaccination strategy it would require a very long time and a large amount of vaccine to achieve the goal. In contrast with allocating the same vaccine amount every year, we find that a variable vaccination strategy with more vaccine given in the beginning followed by less vaccine in later years can save time and total vaccine amount. The variable vaccination strategy illustrated in this study can help to better distribute the vaccine to reduce the HPV prevalence. Although this work is for HPV infection and the case study is for a province in China, the model, analysis and conclusions may be applicable to other sexually transmitted diseases in other regions or countries.
\end{abstract}

Keywords: HPV, two-sex, deterministic model, vaccination strategy, sexually transmitted diseases

*Correspondence should be addressed to M. Martcheva (maia@ufl.edu) or L. Rong (libinrong@ufl.edu). 


\section{Introduction}

Human papillomavirus (HPV) is mainly transmitted through sexual contact. There are more than 100 types of HPV, among which at least 14 can cause cancer and are known as high risk types. Almost all sexually active people are infected at some point in their lives and some may be repeatedly infected. HPV infections usually clear up without any intervention within a few months after acquisition, and about $90 \%$ will clear within 2 years. However, in some cases, HPV infection can persist and progress to cancer [1]. Cervical cancer is the most common HPV-related cancer in women, with an estimated 569,847 new cases and 311,365 deaths in 2018 globally. There is some evidence linking HPV infection with other cancers of vulva, anus, vagina, penis and oropharynx. In the high risk type group, HPV 16/18 cause $70 \%$ of cervical cancers and pre-cancerous cervical lesions. In the low risk type family, HPV 6/11 result in $90 \%$ of genital warts and most RRP (recurrent respiratory papillomatosis) [1].

Vaccination is an effective way to prevent HPV infection. There are currently 3 prophylactic vaccines available. The bivalent, quadrivalent and 9-valent vaccine protect people from HPV types $16 / 18,16 / 18 / 6 / 11$ and 16/18/6/11/31/33/45/52/58, respectively. HPV vaccines have been shown to be safe and very effective in preventing HPV infection and its sequelae [2,3]. HPV vaccine works more effectively if injected before potential exposure to HPV. Therefore, the World Health Organization (WHO) recommends to vaccinate girls aged 9-14 [1]. By October 2019, more than 100 countries have introduced HPV vaccines to their national schedules [4]. In many countries, HPV vaccines are only offered to pre-adolescent girls (may also include catch-up programs for older females). An increasing number of countries such as Australia, the US and UK also recommend HPV vaccines to pre-adolescent boys and young men, including men who have sex with men (MSM) [5-7]. However, due to the shortage of HPV vaccines, the WHO has called for countries to suspend vaccination of boys in December, 2019 [8]. There has been a long-standing debate about offering HPV vaccines to boys. Without vaccinating boys, health benefits brought from vaccine would not be maximized [9]. However, a few transmission dynamic models showed that strong herd effects were expected from girls-only policy, even with coverage as low as $20 \%$ [10]. Should boys be included in a vaccination program? How to allocate HPV vaccines available in a place to maximize the benefit? These questions need to be further investigated.

During the past two decades, a number of mathematical models have been developed to study the epidemiological and economic consequences of HPV vaccination. Elbasha et al. constructed a dynamic model including both demographic and epidemiologic components to assess the epidemiologic consequences and cost-effectiveness of quadrivalent HPV vaccination strategies [11]. They found that vaccinating girls and women was cost-effective and including men and boys was the most effective strategy. Using a similar model, they showed that the quadrivalent HPV vaccines would be cost-effective when administered to females aged 12-24 years or to both females and males before age 12 with a 12-24 years of age catch-up program [12]. By updating the above two models, Elbasha and his collaborator concluded that expanding the current quadrivalent HPV vaccines to boys and men aged 9-26 could provide tremendous public health benefits and was also cost-effective in the United States [13]. 
The models from ref. [11-13] provided a framework, on which more mathematical model have been developed to study HPV infection and vaccination [14-20]. For example, Insinga et al. found the most effective strategy for quadrivalent HPV vaccines in Mexico was vaccinating 12 years old children plus a temporary 12-24 years old catch-up program covering both sexes [14]. Genderneutral program was also considered to be a cost-effective choice in France and Italy [17, 21]. On the other hand, there are several studies concluding that girls-only program is more cost-effective [16, 22-25]. For instance, Cody et al. compared different vaccination strategies of 4-valent or 9valent HPV vaccines in Japan. They found that the most cost-effective strategy was the vaccination program with 9-valent vaccine targeting 12-16 years old girls together with a temporary catch-up program [16]. Similarly, Kim et al. showed that increasing the coverage in girls was more effective and less costly than including boys in a low-resource setting [22]. Damm et al. found that the cost-effectiveness of additional vaccination for boys was highly dependent on the coverage in girls [26].

Some other papers investigated the impact of HPV vaccines and proper vaccine distributions without considering cost-effectiveness [27-45]. Several of them suggested that girls-only policy was better than including boys, at least in the present situation [27-31]. Vaccinating boys was only reasonable if the vaccination coverage for girls was moderate or high [33]. Brisson et al. found that the benefit of vaccinating boys decreased as the coverage in girls increased [30]. However, Azevedo et al. showed that without including men in a vaccination program the disease could only be controlled when more than $90 \%$ of women were vaccinated [32]. Muñoz-Quiles et al. constructed a computational network model and found that HPV-related diseases in women would be eliminated within five decades if the vaccine coverage can achieve $75 \%$ for both females and males [44]. In addition, Bogaards et al. found that giving vaccines to the gender with the highest prevaccine prevalence would most reduce the prevalence [28]. Waning immunity was also considered to be important, especially when studying persistent HPV infection and its associated cancer incidence $[31,34]$. The impact of HPV vaccination was also evaluated in the population of MSM [27, 44-46]. Díez-Domingo et al. showed that MSM would not benefit by the herd immunity effect of vaccinating females [46]. In our previous paper [27], we found that the heterosexual population gets great benefit but MSM only get minor benefit from vaccinating heterosexual females or males. The priority of vaccination should be given to MSM in order to eliminate HPV infection, especially in places that have already achieved high coverage in females.

Most of the above studies obtained the results on vaccine distribution either from cost-effectiveness analysis or numerical simulations, without providing analytical results. In this paper, we will use a two-sex deterministic model to analytically investigate the vaccine distribution strategy. Using the data from Guangxi Province in China as a case study, we will study what strategies would save time and the total amount of vaccines. Specifically, we will mainly address the following questions: 1 . Given a fixed vaccine amount, what is the best way to split the vaccines between the two genders to reduce HPV prevalence? 2. In the case study, what is the threshold of vaccine amount needed to eliminate HPV infection? 3. To reduce HPV prevalence to below a certain threshold, how many years and how many total vaccines are needed under different strategies? How to best allocate these vaccines? To answer these questions, we will formulate and analyze the model in Section 2 and 
3 , respectively. We conduct a variety of simulations on different vaccine distribution strategies in Section 4. Some discussions of the results follow in Section 5.

\section{Model formulation}

In this section, we formulate a two-sex deterministic model to study the transmission of HPV infection in a heterosexually active population. The population is divided into two groups, namely, heterosexual females and heterosexual males (for simplicity, we will use females and males below), and subscripts $f$ and $m$ are used to denote them. In each group, the population is divided into 3 classes: susceptible $\left(S_{k}\right)$, vaccinated $\left(V_{k}\right)$ and infected individuals $\left(I_{k}\right)$, where $k \in\{f, m\}$.

In the absence of vaccination, we assume that humans become sexually active and enter the susceptible compartment $S_{k}$ with the recruitment rate $\Lambda_{k}$. They leave a compartment at a rate $\mu_{k}$. Susceptible individuals are infected by HPV with the force of infection $\lambda_{k}$. Upon infection, the host moves to the infected compartment $I_{k}$. Infected people can clear infection at a rate $\delta_{k}$. Although natural recovery can provide protection against future infection for many other virus infections, the situation for HPV might be different. Several studies found that HPV reinfection is common for both females and males, even with the same HPV type [47, 48]. Therefore, in this paper we formulate a deterministic model based on the SIS (susceptible-infected-susceptible) structure, which was also used in some other HPV modeling studies such as ref. [25, 37]. We will discuss the potential influence of adopting a different model, e.g. SIR (susceptible-infected-recovered), on our results.

In the model with vaccination, we assume that a fraction $\left(\phi_{k}\right)$ of susceptibles are vaccinated and vaccine-induced immunity does not wane during the sexually active period. Vaccine offers a degree of protection $\tau(0 \leq \tau \leq 1)$ regardless of gender. Thus, the probability of a vaccinated person getting infected and moving to the infected compartment $I_{k}$ is $1-\tau$. We also assume that all infected individuals, vaccinated or not, can clear infection and become susceptible at a rate $\delta_{k}$. The model is described by the following system of ordinary differential equations. A schematic diagram of the model is shown in Figure 1.

$$
\left\{\begin{array}{l}
S_{k}^{\prime}(t)=\left(1-\phi_{k}\right) \Lambda_{k}-\lambda_{k} S_{k}+\delta_{k} I_{k}-\mu_{k} S_{k} \\
V_{k}^{\prime}(t)=\phi_{k} \Lambda_{k}-(1-\tau) \lambda_{k} V_{k}-\mu_{k} V_{k} \\
I_{k}^{\prime}(t)=\lambda_{k}\left[S_{k}+(1-\tau) V_{k}\right]-\left(\delta_{k}+\mu_{k}\right) I_{k}
\end{array}\right.
$$

The force of infection is given by

$$
\lambda_{k}=\frac{\beta_{k^{\prime} k} I_{k^{\prime}}}{N_{k}}
$$

where $N_{k}=S_{k}+V_{k}+I_{k}, k, k^{\prime} \in\{f, m\}$ and $k \neq k^{\prime}$. The total population is $N=N_{f}+N_{m}$.

Taking the sum of $S_{k}, V_{k}$ and $I_{k}$ in system (1), we get $N_{k}^{\prime}=\Lambda_{k}-\mu_{k} N_{k}, k=f, m$. Thus, the equilibrium of $N_{k}$ is $\Lambda_{k} / \mu_{k}$. We define the domain of the system (1) to be

$$
D=\left\{\left(S_{f}, V_{f}, I_{f}, S_{m}, V_{m}, I_{m}\right) \in \mathfrak{R}_{+}^{6}: S_{k}+V_{k}+I_{k} \leq \Lambda_{k} / \mu_{k}, k=f, m\right\} .
$$


Using a similar method in the previous study [27], we can verify that $D$ is positively invariant for system (1) and the model is both epidemiologically and mathematically well posed.

\section{Analysis of the model}

\subsection{The model without vaccination}

The model (1) without vaccination reduces to

$$
\left\{\begin{array}{l}
S_{k}^{\prime}(t)=\Lambda_{k}-\lambda_{k} S_{k}+\delta_{k} I_{k}-\mu_{k} S_{k} \\
I_{k}^{\prime}(t)=\lambda_{k} S_{k}-\left(\delta_{k}+\mu_{k}\right) I_{k}
\end{array}\right.
$$

The force of infection is given by

$$
\lambda_{k}=\frac{\beta_{k^{\prime} k} I_{k^{\prime}}}{N_{k}},
$$

where $N_{k}=S_{k}+I_{k}$, with $k, k^{\prime} \in\{f, m\}$ and $k \neq k^{\prime}$.

We define

$$
R_{0, m f}=\frac{\beta_{m f}}{\delta_{m}+\mu_{m}}, \quad R_{0, f m}=\frac{\beta_{f m}}{\delta_{f}+\mu_{f}} .
$$

$R_{0, m f}$ represents the number of secondary female infections generated by one infectious male in an entirely susceptible female population during his whole infectious period. $R_{0, f m}$ has similar meaning. Using the next generation approach [49], we derive the basic reproduction number to be

$$
R_{0}=\sqrt{R_{0, m f} R_{0, f m}} .
$$

It represents the number of secondary infections generated by one infectious individual in an entirely susceptible population during the whole infectious period of the individual.

The system (2) always has a disease-free equilibrium (DFE) $E^{0}=\left(\Lambda_{f} / \mu_{f}, 0, \Lambda_{m} / \mu_{m}, 0\right)$. Its local stability is stated in Theorem 1 . We present the global stability of the limiting system in Theorem 2. Their proofs are given in Appendix A and B, respectively.

Theorem 1. When $R_{0}<1$, the DFE $E^{0}$ is locally asymptotically stable; when $R_{0}>1$, the DFE $E^{0}$ is unstable.

Theorem 2. When $R_{0} \leq 1$, the DFE $E^{0}$ is globally asymptotically stable.

By setting the right-hand side of system (2) to zero, we can solve for the endemic equilibrium, which is shown in Theorem 3. Its local stability is stated in Theorem 4. The global stability of the limiting system is given in Theorem 5. The proofs of Theorem 4 and 5 are given in Appendix C and $\mathrm{D}$, respectively.

Theorem 3. When $R_{0}>1$, there exists a unique endemic equilibrium $E^{*}=\left(S_{f}^{*}, I_{f}^{*}, S_{m}^{*}, I_{m}^{*}\right)$, where

$$
S_{k}^{*}=\frac{N_{k^{\prime}}^{*}\left(\delta_{k^{\prime}}+\mu_{k^{\prime}}\right)+N_{k}^{*} \beta_{k k^{\prime}}}{d_{k} N_{k^{\prime}}^{*}\left(\delta_{k^{\prime}}+\mu_{k^{\prime}}\right)}, \quad I_{k}^{*}=\frac{R_{0}^{2}-1}{d_{k}}, \quad d_{k}=\frac{R_{0}^{2}}{N_{k}^{*}}+\frac{\beta_{k k^{\prime}}}{N_{k^{\prime}}^{*}\left(\delta_{k^{\prime}}+\mu_{k^{\prime}}\right)},
$$


with $N_{k}^{*}=\Lambda_{k} / \mu_{k}, k, k^{\prime} \in\{f, m\}, k \neq k^{\prime}$.

Theorem 4 . When $R_{0}>1$, the endemic equilibrium $E^{*}$ is locally asymptotically stable.

Theorem 5. When $R_{0}>1$, the endemic equilibrium $E^{*}$ is globally asymptotically stable.

\subsection{The model with vaccination: Analysis and best vaccination strategy}

In this section, we study model (1) with vaccination. Similar to the model without vaccination, we define

$$
\begin{aligned}
& R_{0, m f}\left(\phi_{f}\right)=\frac{\beta_{m f}}{\delta_{m}+\mu_{m}}\left[\left(1-\phi_{f}\right)+(1-\tau) \phi_{f}\right], \\
& R_{0, f m}\left(\phi_{m}\right)=\frac{\beta_{f m}}{\delta_{f}+\mu_{f}}\left[\left(1-\phi_{m}\right)+(1-\tau) \phi_{m}\right] .
\end{aligned}
$$

Using the next generation approach, we derive the basic reproduction number to be

$$
R_{0}\left(\phi_{f}, \phi_{m}\right)=\sqrt{R_{0, m f}\left(\phi_{f}\right) R_{0, f m}\left(\phi_{m}\right)}
$$

When $\phi_{f}=\phi_{m}=0$, it is the same as the basic reproduction number for the model without vaccination.

The system (1) always has a disease-free equilibrium

$$
E^{0}=\left(\left(1-\phi_{f}\right) \frac{\Lambda_{f}}{\mu_{f}}, \phi_{f} \frac{\Lambda_{f}}{\mu_{f}}, 0,\left(1-\phi_{m}\right) \frac{\Lambda_{m}}{\mu_{m}}, \phi_{m} \frac{\Lambda_{m}}{\mu_{m}}, 0\right) .
$$

Its local stability is stated in Theorem 6. The proof is similar to Theorem 1 and is omitted. Due to the complexity of the model, it is hard to study the global stability for the DFE. However, using a similar method as in ref. [27], we can show that there is no backward bifurcation for system (1). For the endemic equilibrium, we have the result for its existence, which is stated in Theorem 7 and proved in Appendix E.

Theorem 6. When $R_{0}\left(\phi_{f}, \phi_{m}\right)<1$, the DFE $E^{0}$ is locally asymptotically stable; when $R_{0}\left(\phi_{f}, \phi_{m}\right)>$ 1 , the DFE $E^{0}$ is unstable.

Theorem 7. When $R_{0}\left(\phi_{f}, \phi_{m}\right)>1$, the endemic equilibrium exists.

From the above analysis, we know that the condition $R_{0}<1$ is critical for disease elimination. $R_{0}$ is also an important indicator that quantifies how fast the disease spreads. Therefore, given a fixed vaccine amount $v$, we investigate the following optimization problem

$$
\left\{\begin{array}{l}
\operatorname{minimize} R_{0}\left(\phi_{f}, \phi_{m}\right) \\
\text { subject to } 0 \leq \phi_{f} \leq 1,0 \leq \phi_{m} \leq 1, \phi_{f} \Lambda_{f}+\phi_{m} \Lambda_{m}=v
\end{array}\right.
$$

where $0 \leq v \leq \Lambda_{f}+\Lambda_{m}$. The result is stated in the following Theorem and the proof is given in Appendix F. 
Theorem 8. (i) If $\Lambda_{k} \leq \Lambda_{k^{\prime}}$, then $\min R_{0}\left(\phi_{f}, \phi_{m}\right)$ is attained at $\phi_{k}=\frac{v}{\Lambda_{k}}, \phi_{k^{\prime}}=0$ when $v \leq \Lambda_{k}$ and $\phi_{k}=1, \phi_{k^{\prime}}=\frac{v-\Lambda_{k}}{\Lambda_{k}^{\prime}}$ when $v>\Lambda_{k}$. Here $k, k^{\prime} \in\{f, m\}$ and $k \neq k^{\prime}$.

(ii) When $\Lambda_{f}=\Lambda_{m}=\Lambda$, the smaller $\left|\phi_{f}-\phi_{m}\right|$, the bigger $R_{0}\left(\phi_{f}, \phi_{m}\right)$. An even distribution (i.e. $\left.\phi_{f}=\phi_{m}=\frac{v}{2 \Lambda}\right)$ leads to $\max R_{0}\left(\phi_{f}, \phi_{m}\right)$.

The above Theorem shows that to minimize the basic reproduction number $R_{0}$, the gender with a smaller recruit rate should be vaccinated with priority. If there is vaccine left, it will be given to the other gender. When the two genders have the same recruit rate, splitting vaccines evenly is the worst, i.e. resulting in the maximum $R_{0}$. The evener the distribution, the bigger $R_{0}$.

For convenience, we consider $\min R_{0}^{2}$ as a function of $v$ and denote it by $h(v)$. We have the following result with the proof given in Appendix G.

Corollary 1. (i) $h^{\prime}(v)<0$ always holds.

(ii) If $\Lambda_{k} \leq \Lambda_{k^{\prime}},\left|h^{\prime}(v)\right|$ is larger when $v \leq \Lambda_{k}$ than that when $v>\Lambda_{k}$, where $k, k^{\prime} \in\{f, m\}$ and $k \neq k^{\prime}$.

This Corollary shows that as the vaccine amount $v$ increases, the minimum of the basic reproduction number $R_{0}$ decreases. Once the vaccine amount exceeds the smaller recruit rate of the two genders, vaccination becomes less effective in reducing the basic reproduction number.

\section{Vaccination strategies and a case study}

\subsection{Calibration of transmission rates}

In this section, we use the data from Guangxi Province in China to calibrate parameter values used in the model. We will investigate various vaccination strategies on the basis of these parameter values. We determine the transmission rates of HPV 16/18 in the model without vaccination. In 2014, an observational cohort study including 2309 men and 2378 women aged 18-55 was conducted in Liuzhou, Guangxi Province (in the end, 1937 men and 2344 women were included in the analysis). Therefore, we set $\mu_{f}=\mu_{m}=\frac{1}{55-18}$. The median time (95\% CI) to clear HPV $16 / 18$ is $12.3[7.7,13.3]$ months for females and $6.5[6.2,7.7]$ months for males [50]. So we let the recovery rates $(95 \% \mathrm{CI})$ for females and males be $\delta_{f}=12 / 12.3[12 / 13.3,12 / 7.7]$ and $\delta_{m}=12 / 6.5$ [12/7.7, 12/6.2] with unit 1/year, respectively.

The prevalences of HPV 16/18 for females and males are $p_{f}=107 / 2344=4.6 \%$ and $p_{m}=30 / 1937=1.5 \%$, respectively [51]. According to Liuzhou Statistical Yearbook [52], there were 2411020 people aged 18-60 in Liuzhou in 2014, and the total female and male populations are 1817723 and 1961637, respectively. Hence the female and male populations aged 18-55 are estimated as

$$
N_{f}^{*}=2411020 \times \frac{55-18}{60-18} \times \frac{1817723}{1817723+1961637}=1.0216 \times 10^{6}
$$


and

$$
N_{m}^{*}=2411020 \times \frac{55-18}{60-18} \times \frac{1961637}{1817723+1961637}=1.1024 \times 10^{6} .
$$

Since HPV infection had existed in Liuzhou for many years before 2014 and HPV vaccine was available until 2016 in mainland China, we assume that HPV infection was in the endemic state at that time. From the expression of the endemic equilibrium of the model without vaccination, we get

$$
\begin{aligned}
& p_{f}=\frac{I_{f}^{*}}{N_{f}^{*}}=\frac{\frac{\beta_{m f} \beta_{f m}}{\left(\delta_{f}+\mu_{f}\right)\left(\delta_{m}+\mu_{m}\right)}-1}{\frac{\beta_{m f} \beta_{f m}}{\left(\delta_{f}+\mu_{f}\right)\left(\delta_{m}+\mu_{m}\right)}+\frac{N_{f}^{*}}{N_{m}^{*}} \frac{\beta_{f m}}{\delta_{m}+\mu_{m}}}, \\
& p_{m}=\frac{I_{m}^{*}}{N_{m}^{*}}=\frac{\frac{\beta_{m f} \beta_{f m}}{\left(\delta_{f}+\mu_{f}\right)\left(\delta_{m}+\mu_{m}\right)}-1}{\frac{\beta_{m f} \beta_{f m}}{\left(\delta_{f}+\mu_{f}\right)\left(\delta_{m}+\mu_{m}\right)}+\frac{N_{m}^{*}}{N_{f}^{*}} \frac{\beta_{m f}}{\delta_{f}+\mu_{f}}} .
\end{aligned}
$$

With the above values, we get the transmission rates $(95 \% \mathrm{CI})$ from females to males is $\beta_{m f}=$ 2.8693 [2.6594, 4.5373] and from males to females is $\beta_{f m}=0.6967$ [0.5896, 0.7299].

\subsection{Parameter setting}

We apply the model to Guangxi province with a wider range of ages $15-55$. Hence $\mu_{f}=\mu_{m}=$ $\frac{1}{55-15}$. There are two reasons for using a wider age range. One is that some people have sex at young ages (before age 18). The other is that we are interested in the number of target vaccination population (i.e. 14-yea-old children) and want to roughly use it as recruits. The values for $\delta_{f}$, $\delta_{m}, \beta_{m f}$ and $\beta_{f m}$ are the same as above. We estimate the number of 14-year-old boys and girls in Guangxi in 2021-2033 (Figure 2, for details see Appendix H), which are the target vaccination groups. We find that there are only minor changes for both 14-year-old boys and girls from 20212033. Therefore, we use the average number as the recruitment rate, namely, $\Lambda_{f}=340998$ and $\Lambda_{m}=387463$. Applying all these values to the expression of $R_{0}$ without vaccination, we derive that the basic reproduction number for HPV 16/18 within the age group 15-55 in Guangxi is 1.0333. Using type-specific and gender-specific clearance rate and prevalence derived from ref. [50, 51], we get basic reproduction numbers for some other HPV types (Table 2).

Chinese domestic bivalent HPV vaccine has been available in Guangxi since 2020. We apply the bivalent HPV vaccine in our model. The value (range) for vaccine efficacy $\tau$ is 0.899 [0.8170.944] for HPV 16/18 [53]. So far the bivalent HPV vaccine is only available to females in China. Using all the above values in $R_{0}\left(\phi_{f}, \phi_{m}\right)$ and setting $\phi_{m}=0$ and $R_{0}\left(\phi_{f}, \phi_{m}\right)=1$, we calculate the critical value of $\phi_{f}$ for HPV 16/18 elimination is $\hat{\phi}_{f}=0.0705$. The corresponding vaccine amount is $\hat{v}=\hat{\phi}_{f} \Lambda_{f}=24050$.

\subsection{Sensitivity analysis}

We use the partial rank correlation coefficient (PRCC) to evaluate the impact of model parameters on the dynamics of the model (1). The PRCC provides a global sensitivity analysis for nonlinear but monotone relationships between inputs and outputs [54]. From analytical results, 
we know that $R_{0}<1$ is essential to eliminate the disease. Therefore, we are concerned with the parameters that have the greatest impact on $R_{0}$. We are also interested in the parameters that have great impact on the prevalence. Therefore, in our sensitivity analysis, the inputs are parameters and the outputs are $R_{0}$, the prevalence in females, males and the total population. In the inputs, there is a special parameter called dummy, which is introduced to quantify the artifacts (for details see ref. [54]).

In the sensitivity analyses, we choose the vaccination proportion $\phi_{f}=0.08$ and $\phi_{m}=0.01$ as baseline values and [0.01,0.99] as their ranges. Since we only focus on people in the age group 15-55, we fix $\mu_{f}=\mu_{m}=1 /(55-15)$ and use one parameter $\mu$ to represent them. The sample size for $R_{0}$ is 10000 , and for each prevalence is 6000 . The initial condition for the prevalence is pre-vaccination endemic equilibrium, and the end point is 50 years. The negative or positive sign of the PRCC value indicates that the parameter is inversely or positively correlated with the outputs. The parameter with a larger PRCC index (absolute value greater than 0.5) has more significant influence on the output [55]. From Figure 3 and Table 3, we see that the proportions of vaccination $\phi_{f}$ and $\phi_{m}$ always have a great impact on the basic reproduction number and the disease prevalence. This means that vaccination is an effective way to reduce HPV infection. The output is also sensitive to the clearance rates $\delta_{f}$ and $\delta_{m}$. This indicates that infected individuals having an intact immune response or receiving treatment have a better chance to clear the infection. Other sensitive parameters include transmission rates $\beta_{m f}$ and $\beta_{f m}$, which highlights the importance of using condom or other protections to reduce the risk of infection.

\subsection{The best vaccination strategy}

The condition $R_{0}<1$ is critical for disease elimination. We are interested in the minimum vaccine amount needed per year to hit this threshold. Using linear programming, we find that this value is 24050 , which is attained when all vaccines are given to girls (Figure 4(a)(b)). It agrees with the results in Section 4.2. To verify that this is a critical value and giving vaccines to girls is better, we set $v=25000$, which is slightly bigger than the above threshold. We can see $\min R_{0}=0.9987<1$, which is obtained when all vaccines are given to girls (Figure 4(c)). In this case all prevalences in females, males and the total population go to zero. They decline faster than when we give all vaccines to boys or split them evenly (Figure 4(d)-(f)). These results are also consistent with the analysis. More specifically, because $\Lambda_{f}<\Lambda_{m}$ in the case study, vaccinating females firstly results in a smaller $R_{0}$, which leads to a lower prevalence. Interestingly, even for males, vaccinating girls firstly is still better (see Figure 4(e)). This is reasonable because the transmission always involves both genders. Since Figure 4(d)-(f) show that the dynamics of prevalence in females, males and the total population are similar, from now on we will only focus on the prevalence in the total population.

From Figure 4(f) we also notice that although the disease will go extinct eventually, it will take more than 1000 years and the total vaccine needed will be more than $25000 \times 1000=2.5 \times 10^{7}$. A critical question arises: can we find a better vaccination strategy requiring less time and less total vaccine amount? Figure 5 shows some vaccination strategies that need less time and less total 
vaccine amount. Comparing these cases, we estimate that some value $v \in[50000,150000]$ will result in the smallest total vaccine amount.

To further address the above question, we compare the prevalence in the total population for different values of $v$ by vaccinating girls firstly (Figure 6). We set a specific threshold 0.0005 for the prevalence in the total population, and compute the total time and total vaccine amount needed to reach this threshold (Table 4). We find that allocating 60000 vaccine shots per year leads to the smallest total vaccine amount (6205014) among these cases. It will take about 103 years to achieve this goal.

From Figure 6, we also notice that for any fixed vaccine amount $v$, the prevalence decreases at different speed during different time periods. This indicates that the efficacies of reducing the prevalence with the same amount of vaccine are different. Therefore, we consider variable vaccine amount for different time periods. In Figure 7 (a) we consider three vaccination strategies, namely, adjusting vaccine amount every 50 years, every 30 years and every 20 years. We compare them with the fixed vaccine amount $v=60000$ per year. Table 5 shows that all the three variable vaccination strategies need less time and less total vaccine amount.

Furthermore, we consider vaccination with more frequent adjustments. We find strategies with even less time and less total vaccine amount (Table 6). If we allocate 175000 vaccine amount per year in the first 5 years and reduce by 15000 every 5 years, then it only takes about 58 years to achieve our goal and the total vaccine amount is 5225000. If we apply this strategy and consider cross protection of bivalent HPV vaccines for other HPV types, Figure 7 (b) shows the prevalence in the total population for HPV types covered by 9-valent HPV vaccine in the next 60 years.

Now we consider another case in which there are sufficient HPV vaccines. How to reduce the prevalence in the total population as soon as possible? If we only consider vaccinating adolescent girls, which is the main target population recommended by the WHO, the best situation is that all girls are vaccinated before age 15, namely, $\phi_{f}=1$ and $\phi_{m}=0$. In this case, it will take about 27 years to reduce the prevalence in the total population to below 0.0005 (Figure 8 (a)) and the total vaccine amount is 9231805 . For a fixed vaccine amount, although vaccinating girls firstly is better, vaccinating boys is still helpful in reducing the prevalence. For example, if we vaccinate all 14-year-old girls and boys every year, then it will take about 19 years to reduce the prevalence in the total population to below 0.0005 (Figure 8 (a)). However, the total vaccine amount is much higher (13895612) in this case. In practice, it is not realistic to achieve 100\% coverage. Therefore, we consider more cases with different coverages in Table 7 and Figure 8 (a). We see that a higher vaccine coverage in females and males leads to a smaller $R_{0}$ and it takes a shorter time to reduce the prevalence to below 0.0005 .

Catch-up vaccination (i.e. vaccinate older people) is also useful in reducing the prevalence, especially in the beginning when HPV vaccines are introduced. For example, we assume that $\alpha$ is the proportion of women getting catch-up vaccination. Combining with vaccinating all adolescent girls before they become susceptible, the model predicts that the time needed to reduce the prevalence in the total population to below 0.0005 is 20 years when $\alpha=0.02$ and 13 years when $\alpha=0.1$ (Figure 8 (b)). 


\section{Discussion}

In this paper, we developed a two-sex deterministic model to evaluate the epidemiological impact of HPV vaccination in a heterosexually active population. We derived the basic production number $R_{0}$ and studied the stability of equilibria. The analysis shows that $R_{0}$ plays a critical role in predicting how the infection spreads. The smaller $R_{0}$, the lower prevalence in the total population. In order to reduce $R_{0}$, given a fixed vaccine amount, we investigated how to allocate vaccines between the two genders. By rigorous mathematical analysis, we found that $\min R_{0}$ is achieved when vaccinating the gender with a smaller recruit rate firstly and giving the remaining vaccines, if left, to the other gender. This was numerically illustrated by a case study in which there are fewer female recruits in Guangxi. Vaccinating girls firstly results in the smallest $R_{0}$ and lowest prevalence in the total population compared to other strategies. We also considered a special case in which the recruits for females and males are the same. In this case, besides the same conclusion for $\min R_{0}$, we also found allocating vaccines evenly leads to $\max R_{0}$. The evener the distribution, the bigger $R_{0}$, consequently the higher prevalence. We also proved that $\min R_{0}$ decreases as the vaccine amount $v$ increases but vaccination becomes less effective once its amount exceeds the smaller recruit rate.

The above conclusion on vaccine distribution is reasonable because given a fixed vaccine amount, vaccinating the gender that has a smaller recruit rate enables a larger proportion of the population of that gender getting vaccinated. This leads to more reduction in $R_{0}$ and the prevalence in the total population. In a previous paper studying vaccination in a heterosexual population and MSM [27], we assumed the same recruit rate for heterosexual females and males. We found that vaccinating either gender firstly leads to the same prevalence in the total population and this prevalence is lower than splitting vaccines evenly. This agrees with the conclusion here. Bogaards et al. found that vaccinating the gender with a higher pre-vaccination prevalence would result in a larger reduction of the population prevalence [28]. This is also consistent with our result as a higher pre-vaccination prevalence means more people had already gained immunity. Thus, keeping vaccinating that gender will result in a larger proportion of people getting protected. Some other papers also suggested that increasing the vaccine coverage in girls was better than including boys [29-31]. In other words, they also aimed to get a bigger proportion of people protected within the same gender. This strategy indicates that high coverage in one gender can provide strong herd immunity for sexually transmitted diseases, which also agrees with the results in refs. [44, 45].

Using data from Liuzhou, a city in Guangxi province, we calibrated the transmission rates $\beta_{m f}$ and $\beta_{m f}$. We calculated the basic reproduction number $R_{0}=1.0333$ for HPV $16 / 18$ in people aged 15-55. Using the estimated recruits in Guangxi, we predicted that the minimum number of bivalent HPV vaccine shots is 24050 , which should be given to girls. We also derived basic reproduction numbers for some other HPV types. There are few studies in the literature providing estimates of the basic reproduction number for other HPV types. Riesen et al. estimated that $R_{0}$ for HPV 16 in Switzerland was 1.29 [56], and Ribassin-Majed et al. calculated $R_{0}$ for HPV 6/11 in France to be 1.04 [37]. The difference in these estimates could be caused by different assumptions. For example, we adopted the gender-specific clearance rate from data, and the other two models used 
the same clearance rate for both genders. Social and sexual behaviors may also affect the disease spread. Ziyadi et al. obtained a very small basic reproduction number $\left(R_{0}=0.2346\right)$ for the African American population [38]. One possible explanation is that the authors used the fitted recruitment and relatively small infection rates.

Although HPV infection is predicted to go extinct once the vaccine amount exceeds a critical value, it would take a very long time and the total vaccine amount could be huge. To find a better vaccination strategy, namely, within a shorter period of time and with less total vaccine amount, we set a specific goal, i.e., the prevalence in the total population is less than $0.05 \%$. We compared several strategies and found that the one offering 60000 vaccine shots to girls every year resulted in the smallest total vaccine amount. In this case, the total vaccine amount was 6205014 and it would take about 103 years to achieve the goal. In addition, we found that the efficacy for the same amount of vaccine was different during different periods. Therefore, the variable vaccination strategies were further studied. We compared several cases, among which the best one was giving 175000 vaccine shots to girls per year in the first 5 years, followed by reducing by 15000 every 5 years. It only took about 58 years to achieve the goal and the total vaccine amount was 5225000 . Based on these simulations, it is better to allocate more vaccines at the beginning, and then gradually reduce vaccine shots. Similar results were found in another work [25]. They suggested that vaccination should be applied at the maximum level and after approximately half of the time interval, the rate of vaccination should be gradually reduced, reaching zero in the end. In comparison with the continuous optimal control in [25], our work offered some discrete-time control strategies, which would be easier to implement.

In this paper, we employed a simple two-sex deterministic model to evaluate the epidemiological influence of HPV vaccination and used Guangxi as a case study. The model can be extended to take into account more factors, such as age, sexual behavior and some other heterogeneous mixing. In particular, models with age structure would be more realistic to study the spread of sexually transmitted diseases such as HPV infection and its associated diseases due to the change of sexual behavior with age $[44,45]$. We have formulated two such models with age structure to study the dynamics of HPV-associated oropharyngeal cancer and cervical cancer in Texas [19, 20]. The models divide the population into 23 age groups, which is challenging, if not impossible, to obtain any formal analytical results. More comprehensive models would also require more data for parameterization. Here we used the model with a minimum number of parameters that can be calibrated by the case study, performed both analytical and numerical investigations, and provided some quantitative information on the vaccine distribution strategies.

Vaccination of the MSM population might be crucial for disease elimination. Using a model based on a network paradigm and data from Spain, Díez-Domingo et al. found that the MSM group only benefits from a vaccination program that includes males [46]. From both the analysis and numerical investigations in the ref. [27], we showed that in order to eliminate HPV infection, the priority of vaccination should be given to MSM. Because MSM only account for a small portion of the total population, in this paper we focused on the heterosexual population and studied how to allocate HPV vaccine among them. Analysis of the model without MSM provides some analytical results on the vaccine distribution (Theorem 8 and Corollary 1), which are difficult for the full 
model with MSM. When the MSM population is included, numerical results would suggest similar predictions as in the previous paper [27]. For example, the priority of vaccination should be given to MSM for disease elimination. The heterosexual population gets great benefit but MSM only get minor benefit from vaccinating heterosexual females or males. The best vaccination strategy is to vaccinate MSM firstly as many as possible, then distribute the remaining to the heterosexual population.

We used the SIS model to study the vaccination strategies in view of HPV reinfection after recovery in both males and females [47, 48]. If we use the SIR or SIRS model, the basic reproduction number $R_{0}$ remains the same and the prevalences of the male, female and total population show similar dynamics. Therefore, our conclusions are not affected by the choice of these models. In addition, we used the HPV prevalence as a criterion in evaluating various vaccination strategies. If the objective is to reduce HPV-associated diseases such as cervical or oropharyngeal cancer, then the progression from persistent HPV infection to these cancers should be incorporated into models $[19,20,31,57-59]$ and the guideline of vaccination might be different from that informed by this study. Lastly, the parameters and predictions are based on a case study in Guangxi Province in China. This can be applied to other countries or regions. The vaccination strategies obtained in this study may also be applicable to other sexually transmitted diseases.

\section{Appendix A - Proof of Theorem 1}

Reordering variables as $x=\left(I_{f}, I_{m}, S_{f}, S_{m}\right)^{T}$, the Jacobian matrix of system (2) evaluated at the disease-free equilibrium $E^{0}=\left(0,0, S_{f}^{0}, S_{m}^{0}\right)=\left(0,0, \Lambda_{f} / \mu_{f}, \Lambda_{m} / \mu_{m}\right)$ is

$$
J\left(E^{0}\right)=\left(\begin{array}{cc}
J_{11} & 0 \\
J_{21} & J_{22}
\end{array}\right)
$$

where

$$
J_{11}=\left(\begin{array}{cc}
-\left(\delta_{f}+\mu_{f}\right) & \beta_{m f} \\
\beta_{f m} & -\left(\delta_{m}+\mu_{m}\right)
\end{array}\right)
$$

and

$$
J_{22}=\left(\begin{array}{cc}
-\mu_{f} & 0 \\
0 & -\mu_{m}
\end{array}\right)
$$

Clearly, $-\mu_{f}$ and $-\mu_{m}$ are negative eigenvalues of $J\left(E^{0}\right)$. The remaining eigenvalues are determined by the matrix $J_{11}$. If $R_{0}<1$, then we have $\operatorname{Tr}\left(J_{11}\right)<0$ and $\operatorname{det}\left(J_{11}\right)=\left(\delta_{f}+\mu_{f}\right)\left(\delta_{m}+\mu_{m}\right)\left(1-R_{0}^{2}\right)>0$. By the Routh-Hurwitz criterion [60], the DFE $E^{0}$ is locally asymptotically stable.

If $R_{0}>1$, then $\operatorname{det}\left(J_{11}\right)<0$. Hence $J_{11}$ has an eigenvalue with positive real part. This shows that $E^{0}$ is unstable. 


\section{Appendix B - Proof of Theorem 2}

Considering the limiting system of (2), i.e. when $N_{f} \equiv \frac{\Lambda_{f}}{\mu_{f}}$ and $N_{m} \equiv \frac{\Lambda_{m}}{\mu_{m}}$, we define the following Lyapunov function

$$
L=\left(S_{f}-S_{f}^{0}-S_{f}^{0} \ln \frac{S_{f}}{S_{f}^{0}}+I_{f}\right)+R_{0, m f}\left(S_{m}-S_{m}^{0}-S_{m}^{0} \ln \frac{S_{m}}{S_{m}^{0}}+I_{m}\right),
$$

where $S_{k}^{0}=\frac{\Lambda_{k}}{\mu_{k}}, k=f, m$. It is clear that $L$ is radially unbounded and positive definite in the entire space $D$. The derivative of $L$ along the trajectories of system (2) yields

$$
\begin{aligned}
\dot{L}= & {\left[\left(1-\frac{S_{f}^{0}}{S_{f}}\right) S_{f}^{\prime}+I_{f}^{\prime}\right]+R_{0, m f}\left[\left(1-\frac{S_{m}^{0}}{S_{m}}\right) S_{m}^{\prime}+I_{m}^{\prime}\right] } \\
= & \left(1-\frac{S_{f}^{0}}{S_{f}}\right)\left(\Lambda_{f}-\lambda_{f} S_{f}+\delta_{f} I_{f}-\mu_{f} S_{f}\right)+\left[\lambda_{f} S_{f}-\left(\delta_{f}+\mu_{f}\right) I_{f}\right] \\
& +R_{0, m f}\left\{\left(1-\frac{S_{m}^{0}}{S_{m}}\right)\left(\Lambda_{m}-\lambda_{m} S_{m}+\delta_{m} I_{m}-\mu_{m} S_{m}\right)+\left[\lambda_{m} S_{m}-\left(\delta_{m}+\mu_{m}\right) I_{m}\right]\right\} .
\end{aligned}
$$

Using the equilibrium conditions $\Lambda_{k}=\mu_{k} S_{k}^{0}, N_{k}=S_{k}^{0}, \frac{S_{k}^{0}}{S_{k}} \geq 1, k \in\{f, m\}$ and collecting terms, we obtain

$$
\begin{aligned}
\dot{L}= & {\left[\left(1-\frac{S_{f}^{0}}{S_{f}}\right) \mu_{f}\left(S_{f}^{0}-S_{f}\right)+S_{f}^{0} \lambda_{f}-\left(\frac{S_{f}^{0}}{S_{f}} \delta_{f}+\mu_{f}\right) I_{f}\right] } \\
& +R_{0, m f}\left[\left(1-\frac{S_{m}^{0}}{S_{m}}\right) \mu_{m}\left(S_{m}^{0}-S_{m}\right)+S_{m}^{0} \lambda_{m}-\left(\frac{S_{m}^{0}}{S_{m}} \delta_{m}+\mu_{m}\right) I_{m}\right] \\
\leq & {\left[-\frac{\mu_{f}}{S_{f}}\left(S_{f}^{0}-S_{f}\right)^{2}+\beta_{m f} I_{m}-\left(\delta_{f}+\mu_{f}\right) I_{f}\right]+R_{0, m f}\left[-\frac{\mu_{m}}{S_{m}}\left(S_{m}^{0}-S_{m}\right)^{2}+\beta_{f m} I_{f}-\left(\delta_{m}+\mu_{m}\right) I_{m}\right] } \\
= & -\frac{\mu_{f}}{S_{f}}\left(S_{f}^{0}-S_{f}\right)^{2}-R_{0, m f} \frac{\mu_{m}}{S_{m}}\left(S_{m}^{0}-S_{m}\right)^{2}+\left[\beta_{m f}-R_{0, m f}\left(\delta_{m}+\mu_{m}\right)\right] I_{m}+\left[R_{0, m f} \beta_{f m}-\left(\delta_{f}+\mu_{f}\right)\right] I_{f} \\
= & -\frac{\mu_{f}}{S_{f}}\left(S_{f}^{0}-S_{f}\right)^{2}-R_{0, m f} \frac{\mu_{m}}{S_{m}}\left(S_{m}^{0}-S_{m}\right)^{2}+\left(\delta_{f}+\mu_{f}\right)\left(R_{0}^{2}-1\right) I_{f} .
\end{aligned}
$$
is

When $R_{0}<1$, we have that $\dot{L} \leq 0$ and it is equal to 0 only at the DFE. Therefore, by KrasovkiiLaSalle Theorem [61], the DFE $E^{0}$ is globally asymptotically stable when $R_{0} \leq 1$.

\section{Appendix C - Proof of Theorem 4}

The Jacobian matrix of system (2) evaluated at the endemic equilibrium $E^{*}=\left(I_{f}^{*}, I_{m}^{*}, S_{f}^{*}, S_{m}^{*}\right)$

$$
J\left(E^{*}\right)=\left(\begin{array}{cccc}
a_{11} & a_{12} & a_{13} & 0 \\
a_{21} & a_{22} & 0 & a_{24} \\
a_{31} & a_{32} & a_{33} & 0 \\
a_{41} & a_{42} & 0 & a_{44}
\end{array}\right),
$$


where

$$
\begin{aligned}
& a_{11}=-\frac{\beta_{m f} I_{m}^{*} S_{f}^{*}}{N_{f}^{* 2}}-\left(\delta_{f}+\mu_{f}\right) \quad a_{12}=\frac{\beta_{m f} S_{f}^{*}}{N_{f}^{*}} \quad a_{13}=\frac{\beta_{m f} I_{m}^{*}}{N_{f}^{*}}-\frac{\beta_{m f} I_{m}^{*} S_{f}^{*}}{N_{f}^{* 2}} \\
& a_{21}=\frac{\beta_{f m} S_{m}^{*}}{N_{m}^{*}} \quad a_{22}=-\frac{\beta_{f m} I_{f}^{*} S_{m}^{*}}{N_{m}^{* 2}}-\left(\delta_{m}+\mu_{m}\right) \quad a_{24}=\frac{\beta_{f m} I_{f}^{*}}{N_{m}^{*}}-\frac{\beta_{f m} I_{f}^{*} S_{m}^{*}}{N_{m}^{* 2}} \\
& a_{31}=\frac{\beta_{m f} I_{m}^{*} S_{f}^{*}}{N_{f}^{* 2}}+\delta_{f} \quad a_{32}=-\frac{\beta_{m f} S_{f}^{*}}{N_{f}^{*}} \quad a_{33}=-\frac{\beta_{m f} I_{m}^{*}}{N_{f}^{*}}+\frac{\beta_{m f} I_{m}^{*} S_{f}^{*}}{N_{f}^{* 2}}-\mu_{f}, \\
& a_{41}=-\frac{\beta_{f m} S_{m}^{*}}{N_{m}^{*}} \quad a_{42}=\frac{\beta_{f m} I_{f}^{*} S_{m}^{*}}{N_{m}^{* 2}}+\delta_{m} \quad a_{44}=-\frac{\beta_{f m} I_{f}^{*}}{N_{m}^{*}}+\frac{\beta_{f m} I_{f}^{*} S_{m}^{*}}{N_{m}^{* 2}}-\mu_{m} .
\end{aligned}
$$

Then we have

$$
\left|J\left(E^{*}\right)-\lambda I\right|=\left|\begin{array}{cccc}
a_{11}-\lambda & a_{12} & a_{13} & 0 \\
a_{21} & a_{22}-\lambda & 0 & a_{24} \\
a_{31} & a_{32} & a_{33}-\lambda & 0 \\
a_{41} & a_{42} & 0 & a_{44}-\lambda
\end{array}\right| .
$$

Adding the first row to the third row, and the second row to the last row, we get

$$
\left|J\left(E^{*}\right)-\lambda I\right|=\left|\begin{array}{cccc}
a_{11}-\lambda & a_{12} & a_{13} & 0 \\
a_{21} & a_{22}-\lambda & 0 & a_{24} \\
-\mu_{f}-\lambda & 0 & -\mu_{f}-\lambda & 0 \\
0 & -\mu_{m}-\lambda & 0 & -\mu_{m}-\lambda
\end{array}\right| .
$$

The characteristic equation is

$$
\left(\lambda+\mu_{f}\right)\left(\lambda+\mu_{m}\right)\left(\lambda^{2}+b \lambda+c\right)=0
$$

where

$$
\begin{aligned}
& b=\delta_{f}+\mu_{f}+\delta_{m}+\mu_{m}+\frac{\beta_{f m} I_{f}^{*}}{N_{m}^{*}}+\frac{\beta_{m f} I_{m}^{*}}{N_{f}^{*}}>0, \\
& c=\left(\delta_{f}+\mu_{f}\right)\left(\delta_{m}+\mu_{m}\right)+\left(\delta_{f}+\mu_{f}\right) \frac{\beta_{f m} I_{f}^{*}}{N_{m}^{*}}+\left(\delta_{m}+\mu_{m}\right) \frac{\beta_{m f} I_{m}^{*}}{N_{f}^{*}}>0 .
\end{aligned}
$$

Clearly, all eigenvalues of $J\left(E^{*}\right)$ have negative real parts. Therefore, the endemic equilibrium 


\section{Appendix D - Proof of Theorem 5}

Considering limiting system, namely, $N_{f} \equiv N_{f}^{*}=\frac{\Lambda_{f}}{\mu_{f}}$ and $N_{m} \equiv N_{m}^{*}=\frac{\Lambda_{m}}{\mu_{m}}$, system (2) can be reduced to

$$
\left\{\begin{array}{l}
I_{f}^{\prime}=\frac{\beta_{m f} I_{m}\left(N_{f}^{*}-I_{f}\right)}{N_{f}^{*}}-\left(\delta_{f}+\mu_{f}\right) I_{f}, \\
I_{m}^{\prime}=\frac{\beta_{f m} I_{f}\left(N_{m}^{*}-I_{m}\right)}{N_{m}^{*}}-\left(\delta_{m}+\mu_{m}\right) I_{m} .
\end{array}\right.
$$

This is a two-dimensional system, to which we can apply Dulac's criterion. We define

$$
X=\left\{\left(I_{f}, I_{m}\right) \in \mathfrak{R}_{+}^{2}: I_{k} \leq \Lambda_{k} / \mu_{k}, k=f, m\right\} .
$$

Denote

$$
\begin{gathered}
F\left(I_{f}, I_{m}\right)=\frac{\beta_{m f} I_{m}\left(N_{f}^{*}-I_{f}\right)}{N_{f}^{*}}-\left(\delta_{f}+\mu_{f}\right) I_{f}, \\
G\left(I_{f}, I_{m}\right)=\frac{\beta_{f m} I_{f}\left(N_{m}^{*}-I_{m}\right)}{N_{m}^{*}}-\left(\delta_{m}+\mu_{m}\right) I_{m} .
\end{gathered}
$$

Using 1 as the Dulac multiplier, we get

$$
\frac{\partial F\left(I_{f}, I_{m}\right)}{\partial I_{f}}+\frac{\partial G\left(I_{f}, I_{m}\right)}{\partial I_{m}}=-\frac{\beta_{m f} I_{m}}{N_{f}^{*}}-\left(\delta_{f}+\mu_{f}\right)-\frac{\beta_{f m} I_{f}}{N_{m}^{*}}-\left(\delta_{m}+\mu_{m}\right)<0 .
$$

Therefore, there are no periodic orbits in region $X$ [61]. Since the unique endemic equilibrium $E^{*}$ is locally asymptotically stable when $R_{0}>1$, it is globally asymptotically stable when $R_{0}>1$.

\section{Appendix E - Proof of Theorem 7}

To get the endemic equilibrium, we set the right-hand side of system (1) to zero. At the equilibrium, we use $N_{k}^{*}-V_{k}-I_{k}$ to replace $S_{k}$, where $N_{k}^{*}=\Lambda_{k} / \mu_{k}, k \in\{f, m\}$. It follows that

$$
\left\{\begin{array}{l}
\phi_{f} \Lambda_{f}-(1-\tau) \lambda_{f} V_{f}-\mu_{f} V_{f}=0 \\
\lambda_{f}\left(N_{f}^{*}-\tau V_{f}-I_{f}\right)-\left(\delta_{f}+\mu_{f}\right) I_{f}=0 \\
\phi_{m} \Lambda_{m}-(1-\tau) \lambda_{m} V_{m}-\mu_{m} V_{m}=0 \\
\lambda_{m}\left(N_{m}^{*}-\tau V_{m}-I_{m}\right)-\left(\delta_{m}+\mu_{m}\right) I_{m}=0
\end{array}\right.
$$

where

$$
\lambda_{k}=\frac{\beta_{k^{\prime} k} I_{k^{\prime}}}{N_{k}^{*}}, \quad k, k^{\prime} \in\{f, m\}, \quad k \neq k^{\prime}
$$


From the third equation of (4), we get

$$
V_{m}=\frac{\phi_{m} \Lambda_{m}}{(1-\tau) \lambda_{m}+\mu_{m}}=\frac{\phi_{m} \Lambda_{m}}{(1-\tau) \frac{\beta_{f m} I_{f}}{N_{m}^{*}}+\mu_{m}} \text {, denoted by } g_{1}\left(I_{f}\right) .
$$

From the last equation of (4), we have $I_{m}=g_{2}\left(I_{f}\right) I_{f}$, where

$$
g_{2}\left(I_{f}\right)=\frac{\left[N_{m}^{*}-\tau g_{1}\left(I_{f}\right)\right] \frac{\beta_{f m}}{N_{m}^{*}}}{\frac{\beta_{f m}}{N_{m}^{*}} I_{f}+\delta_{m}+\mu_{m}} .
$$

Substituting into the first equation of (4), we get

$$
V_{f}=\frac{\phi_{f} \Lambda_{f}}{(1-\tau) \lambda_{f}+\mu_{f}}=\frac{\phi_{f} \Lambda_{f}}{(1-\tau) \frac{\beta_{m f} g_{2}\left(I_{f}\right) I_{f}}{N_{f}^{*}}+\mu_{f}} \text {, denoted by } g_{3}\left(I_{f}\right) \text {. }
$$

Substituting into the second equation of (4), we have

$$
\frac{\beta_{m f}}{N_{f}^{*}} g_{2}\left(I_{f}\right) I_{f}\left[N_{f}^{*}-\tau g_{3}\left(I_{f}\right)-I_{f}\right]-\left(\delta_{f}+\mu_{f}\right) I_{f}=0 .
$$

Define

$$
G\left(I_{f}\right)=\frac{\beta_{m f}}{N_{f}^{*}} g_{2}\left(I_{f}\right)\left[N_{f}^{*}-\tau g_{3}\left(I_{f}\right)-I_{f}\right]-\left(\delta_{f}+\mu_{f}\right) .
$$

We notice that

$$
g_{1}(0)=\phi_{m} N_{m}^{*}, \quad g_{2}(0)=\frac{\left(1-\tau \phi_{m}\right) \beta_{f m}}{\delta_{m}+\mu_{m}}, \quad g_{3}(0)=\phi_{f} N_{f}^{*} .
$$

Hence

$$
\begin{aligned}
G(0) & =\frac{\beta_{m f}}{N_{f}^{*}} g_{2}(0)\left[N_{f}^{*}-\tau g_{3}(0)\right]-\left(\delta_{f}+\mu_{f}\right) \\
& =\frac{\beta_{m f}}{N_{f}^{*}} \frac{\left(1-\tau \phi_{m}\right) \beta_{f m}}{\delta_{m}+\mu_{m}}\left[N_{f}^{*}-\tau \phi_{f} N_{f}^{*}\right]-\left(\delta_{f}+\mu_{f}\right) \\
& =\frac{\beta_{m f} \beta_{f m}\left(1-\tau \phi_{m}\right)\left(1-\tau \phi_{f}\right)}{\delta_{m}+\mu_{m}}-\left(\delta_{f}+\mu_{f}\right) \\
& =\left(\delta_{f}+\mu_{f}\right)\left[R_{0}^{2}\left(\phi_{f}, \phi_{m}\right)-1\right] .
\end{aligned}
$$

Thus, $G(0)>0$ when $R_{0}\left(\phi_{f}, \phi_{m}\right)>1$. On the other hand,

$$
\lim _{I_{f} \rightarrow N_{f}^{*}} g_{1}\left(I_{f}\right)=\lim _{I_{f} \rightarrow N_{f}^{*}} \frac{\phi_{m} \Lambda_{m}}{(1-\tau) \frac{\beta_{f m} I_{f}}{N_{m}^{*}}+\mu_{m}}<\frac{\phi_{m} \Lambda_{m}}{\mu_{m}}=\phi_{m} N_{m}^{*} \leq N_{m}^{*} .
$$


Hence, $\lim _{I_{f} \rightarrow N_{f}^{*}}\left[N_{m}^{*}-\tau g_{1}\left(I_{f}\right)\right]>0$. Consequently

$$
\lim _{I_{f} \rightarrow N_{f}^{*}} g_{2}\left(I_{f}\right)=\lim _{I_{f} \rightarrow N_{f}^{*}} \frac{\left[N_{m}^{*}-\tau g_{1}\left(I_{f}\right)\right] \frac{\beta_{f m}}{N_{m}^{*}}}{\frac{\beta_{f m}}{N_{m}^{*}} I_{f}+\delta_{m}+\mu_{m}}>0 .
$$

It follows that

$$
\lim _{I_{f} \rightarrow N_{f}^{*}} g_{3}\left(I_{f}\right)=\lim _{I_{f} \rightarrow N_{f}^{*}} \frac{\phi_{f} \Lambda_{f}}{(1-\tau) \frac{\beta_{m f} g_{2}\left(I_{f}\right) I_{f}}{N_{f}^{*}}+\mu_{f}} \geq 0
$$

Therefore,

$$
\lim _{I_{f} \rightarrow N_{f}^{*}}\left[N_{f}^{*}-\tau g_{3}\left(I_{f}\right)-I_{f}\right] \leq \lim _{I_{f} \rightarrow N_{f}^{*}}\left(N_{f}^{*}-I_{f}\right)=0 .
$$

Since $\lim _{I_{f} \rightarrow N_{f}^{*}} g_{2}\left(I_{f}\right)>0$,

$$
\lim _{I_{f} \rightarrow N_{f}^{*}} G\left(I_{f}\right)=\lim _{I_{f} \rightarrow N_{f}^{*}}\left\{\frac{\beta_{m f}}{N_{f}^{*}} g_{2}\left(I_{f}\right)\left[N_{f}^{*}-\tau g_{3}\left(I_{f}\right)-I_{f}\right]-\left(\delta_{f}+\mu_{f}\right)\right\} \leq-\left(\delta_{f}+\mu_{f}\right)<0 .
$$

Therefore, there exists $I_{f}^{*} \in\left(0, N_{f}^{*}\right)$ such that $G\left(I_{f}^{*}\right)=0$. It follows that $V_{m}^{*}=g_{1}\left(I_{f}^{*}\right) \in\left(0, \phi_{m} N_{m}^{*}\right)$, which indicates $g_{2}\left(I_{f}^{*}\right)>0$. Hence $I_{m}^{*}=g_{2}\left(I_{f}^{*}\right) I_{f}^{*}>0$. Since $\lambda_{m}^{*}=\beta_{f m} I_{f}^{*} / N_{m}^{*}>0$, we have $S_{m}^{*}=\left[\left(1-\phi_{m}\right) \Lambda_{m}+\delta_{m} I_{m}^{*}\right] /\left(\lambda_{m}^{*}+\mu_{m}\right)>0$. Since $S_{m}^{*}+V_{m}^{*}+I_{m}^{*}=N_{m}^{*}$, we have $S_{m}^{*}, V_{m}^{*}, I_{m}^{*} \in\left(0, N_{m}^{*}\right)$. Similarly, we have $S_{f}^{*}, V_{f}^{*}, I_{f}^{*} \in\left(0, N_{f}^{*}\right)$. This completes the proof.

\section{Appendix F - Proof of Theorem 8}

From $\phi_{f} \Lambda_{f}+\phi_{m} \Lambda_{m}=v$, we derive $\phi_{m}=\frac{v-\phi_{f} \Lambda_{f}}{\Lambda_{m}}$. It follows that

$$
\begin{aligned}
R_{0}^{2}\left(\phi_{f}, \phi_{m}\right) & =\frac{\beta_{m f} \beta_{f m}}{\left(\delta_{m}+\mu_{m}\right)\left(\delta_{f}+\mu_{f}\right)}\left(1-\tau \phi_{f}\right)\left(1-\tau \phi_{m}\right) \\
& =\frac{\beta_{m f} \beta_{f m}}{\left(\delta_{m}+\mu_{m}\right)\left(\delta_{f}+\mu_{f}\right)}\left(1-\tau \phi_{f}\right)\left(1-\tau \frac{v-\phi_{f} \Lambda_{f}}{\Lambda_{m}}\right),
\end{aligned}
$$

where

$$
\max \left\{0, \frac{v-\Lambda_{m}}{\Lambda_{f}}\right\} \leq \phi_{f} \leq \min \left\{1, \frac{v}{\Lambda_{f}}\right\}
$$

We have 4 cases.

Case 1. $v \leq \Lambda_{f}$ and $v \leq \Lambda_{m}$.

It is easy to check that

$$
\max \left\{0, \frac{v-\Lambda_{m}}{\Lambda_{f}}\right\}=0, \quad \min \left\{1, \frac{v}{\Lambda_{f}}\right\}=\frac{v}{\Lambda_{f}}
$$


Hence

$$
0 \leq \phi_{f} \leq \frac{v}{\Lambda_{f}}
$$

The value $\min R_{0}^{2}\left(\phi_{f}, \phi_{m}\right)$ can only be attained at point $\phi_{f}=0$ or $\phi_{f}=\frac{v}{\Lambda_{f}}$. Since

$$
\begin{aligned}
& \left.R_{0}^{2}\right|_{\phi_{f}=0}=\frac{\beta_{m f} \beta_{f m}}{\left(\delta_{m}+\mu_{m}\right)\left(\delta_{f}+\mu_{f}\right)}\left(1-\tau \frac{v}{\Lambda_{m}}\right), \\
& \left.R_{0}^{2}\right|_{\phi_{f}=\frac{v}{\Lambda_{f}}}=\frac{\beta_{m f} \beta_{f m}}{\left(\delta_{m}+\mu_{m}\right)\left(\delta_{f}+\mu_{f}\right)}\left(1-\tau \frac{v}{\Lambda_{f}}\right),
\end{aligned}
$$

and $\min R_{0}$ and $\min R_{0}^{2}$ are obtained at the same point, we have the following results:

(a) When $v \leq \Lambda_{m}<\Lambda_{f}$, $\min R_{0}\left(\phi_{f}, \phi_{m}\right)$ is attained at point $\phi_{f}=0, \phi_{m}=\frac{v}{\Lambda_{m}}$;

(b) When $v \leq \Lambda_{f}<\Lambda_{m}, \min R_{0}\left(\phi_{f}, \phi_{m}\right)$ is attained at point $\phi_{f}=\frac{v}{\Lambda_{f}}, \phi_{m}=0$;

(c) When $v \leq \Lambda_{f}=\Lambda_{m}, \min R_{0}\left(\phi_{f}, \phi_{m}\right)$ is attained at point $\phi_{f}=0, \phi_{m}=\frac{v}{\Lambda_{m}}$ or $\phi_{f}=\frac{v}{\Lambda_{f}}, \phi_{m}=0$.

Case 2. $\Lambda_{f}<v \leq \Lambda_{m}$

In this case, we have

$$
\max \left\{0, \frac{v-\Lambda_{m}}{\Lambda_{f}}\right\}=0, \quad \min \left\{1, \frac{v}{\Lambda_{f}}\right\}=1
$$

Thus, $0 \leq \phi_{f} \leq 1$. By the same reason as above, we compare

$$
\left.R_{0}^{2}\right|_{\phi_{f}=0}=\frac{\beta_{m f} \beta_{f m}}{\left(\delta_{m}+\mu_{m}\right)\left(\delta_{f}+\mu_{f}\right)}\left(1-\tau \frac{v}{\Lambda_{m}}\right)
$$

and

$$
\left.R_{0}^{2}\right|_{\phi_{f}=1}=\frac{\beta_{m f} \beta_{f m}}{\left(\delta_{m}+\mu_{m}\right)\left(\delta_{f}+\mu_{f}\right)}(1-\tau)\left(1-\tau \frac{v-\Lambda_{f}}{\Lambda_{m}}\right) .
$$

Since $\Lambda_{f}<v \leq \Lambda_{m}$ and $v \leq \Lambda_{f}+\Lambda_{m}$, we have $1-\tau \leq 1-\tau \frac{v}{\Lambda_{m}}$ and $0 \leq 1-\tau \leq 1-\tau \frac{v-\Lambda_{f}}{\Lambda_{m}}<1$, which implies $\left.R_{0}^{2}\right|_{\phi_{f}=0}>\left.R_{0}^{2}\right|_{\phi_{f}=1}$. Therefore, we have the following result:

(d) When $\Lambda_{f}<v \leq \Lambda_{m}, \min R_{0}\left(\phi_{f}, \phi_{m}\right)$ is attained at point $\phi_{f}=1, \phi_{m}=\frac{v-\Lambda_{f}}{\Lambda_{m}}$.

Case 3. $\Lambda_{m}<v \leq \Lambda_{f}$.

By similar argument as in Case 2, we can derive the following result:

(e) When $\Lambda_{m}<v \leq \Lambda_{f}, \min R_{0}\left(\phi_{f}, \phi_{m}\right)$ is attained at point $\phi_{f}=\frac{v-\Lambda_{m}}{\Lambda_{f}}, \phi_{m}=1$.

Case 4. $v>\Lambda_{f}$ and $v>\Lambda_{m}$.

By similar argument as above, we compare

$$
\left.R_{0}^{2}\right|_{\phi_{f}=\frac{v-\Lambda_{m}}{\Lambda_{f}}}=\frac{\beta_{m f} \beta_{f m}}{\left(\delta_{m}+\mu_{m}\right)\left(\delta_{f}+\mu_{f}\right)}\left(1-\tau \frac{v-\Lambda_{m}}{\Lambda_{f}}\right)(1-\tau)
$$


and

$$
\left.R_{0}^{2}\right|_{\phi_{f}=1}=\frac{\beta_{m f} \beta_{f m}}{\left(\delta_{m}+\mu_{m}\right)\left(\delta_{f}+\mu_{f}\right)}(1-\tau)\left(1-\tau \frac{v-\Lambda_{f}}{\Lambda_{m}}\right)
$$

When $\Lambda_{f}<\Lambda_{m}$, from $v \leq \Lambda_{f}+\Lambda_{m}$ we have $\left(\Lambda_{m}-\Lambda_{f}\right) v \leq \Lambda_{m}^{2}-\Lambda_{f}^{2}$. Hence $\frac{v-\Lambda_{m}}{\Lambda_{f}} \leq \frac{v-\Lambda_{f}}{\Lambda_{m}}$. This implies $\left.R_{0}^{2}\right|_{\phi_{f}=\frac{v-\Lambda_{m}}{\Lambda_{f}}} \geq\left. R_{0}^{2}\right|_{\phi_{f}=1}$. Similarly, when $\Lambda_{f}>\Lambda_{m}$, we can derive $\left.R_{0}^{2}\right|_{\phi_{f}=\frac{v-\Lambda_{m}}{\Lambda_{f}}} \leq\left. R_{0}^{2}\right|_{\phi_{f}=1}$. Therefore, we have the following results:

(f) When $\Lambda_{f}<\Lambda_{m}<v, \min R_{0}\left(\phi_{f}, \phi_{m}\right)$ is attained at point $\phi_{f}=1, \phi_{m}=\frac{v-\Lambda_{f}}{\Lambda_{m}}$;

(g) When $\Lambda_{m}<\Lambda_{f}<v, \min R_{0}\left(\phi_{f}, \phi_{m}\right)$ is attained at point $\phi_{f}=\frac{v-\Lambda_{m}}{\Lambda_{f}}, \phi_{m}=1$;

(h) When $\Lambda_{f}=\Lambda_{m}<v, \min R_{0}\left(\phi_{f}, \phi_{m}\right)$ is attained at point $\phi_{f}=1, \phi_{m}=\frac{v-\Lambda_{f}}{\Lambda_{m}}$ or $\phi_{f}=\frac{v-\Lambda_{m}}{\Lambda_{f}}$, $\phi_{m}=1$.

Summing up all the results from (a)-(h), we get the following results. If $\Lambda_{k} \leq \Lambda_{k^{\prime}}, \min R_{0}\left(\phi_{f}, \phi_{m}\right)$ is attained at: (i) $\phi_{k}=\frac{v}{\Lambda_{k}}, \phi_{k^{\prime}}=0$ when $v \leq \Lambda_{k}$; (ii) $\phi_{k}=1, \phi_{k^{\prime}}=\frac{v-\Lambda_{k}}{\Lambda_{k}^{\prime}}$ when $v>\Lambda_{k}$, where $k, k^{\prime} \in\{f, m\}$ and $k \neq k^{\prime}$.

Considering the special case $\Lambda_{f}=\Lambda_{m}=\Lambda$, we only have Case 1 and Case 4. If we consider $R_{0}^{2}\left(\phi_{f}, \phi_{m}\right)$ as a function of $\phi_{f}$, then it becomes a quadratic function, which has the axis of symmetry $\phi_{f}=\frac{v}{2 \Lambda}$. In either case, from $v \leq 2 \Lambda$ we have $\max \left\{0, \frac{v-\Lambda}{\Lambda}\right\} \leq \phi_{f}^{*}=\frac{v}{2 \Lambda} \leq \min \left\{1, \frac{v}{\Lambda}\right\}$. According to the properties of quadratic functions, $\max R_{0}\left(\phi_{f}, \phi_{m}\right)$ can be achieved at $\phi_{f}^{*}$, and the closer $\phi_{f}$ approaches $\phi_{f}^{*}$, the bigger $R_{0}$ is. Moreover, $\phi_{f}^{*}=\frac{v}{2 \Lambda}$ corresponds to $\phi_{f}^{*}=\phi_{m}^{*}=\frac{v}{2 \Lambda}$. When $\phi_{f}$ approaches $\phi_{f}^{*}, \phi_{f}$ and $\phi_{m}$ are getting closer to each other. In other words, when $\Lambda_{f}=\Lambda_{m}=\Lambda$, $\max R_{0}\left(\phi_{f}, \phi_{m}\right)$ is attained at $\phi_{f}=\phi_{m}=\frac{v}{2 \Lambda}$, and the smaller $\left|\phi_{f}-\phi_{m}\right|$, the bigger $R_{0}\left(\phi_{f}, \phi_{m}\right)$.

\section{Appendix G - Proof of Corollary 1}

Considering $\min R_{0}^{2}$ as a function of the vaccine amount $v$ and denote it by $h(v)$, we can summarize the results from Appendix $\mathrm{F}$ as follows:

(i) When $\Lambda_{f} \leq \Lambda_{m}$

$$
h(v)= \begin{cases}R_{0}^{2}\left(\frac{v}{\Lambda_{f}}, 0\right)=\frac{\beta_{m f} \beta_{f m}}{\left(\delta_{m}+\mu_{m}\right)\left(\delta_{f}+\mu_{f}\right)}\left(1-\tau \frac{v}{\Lambda_{f}}\right), & v \leq \Lambda_{f}, \\ R_{0}^{2}\left(1, \frac{v-\Lambda_{f}}{\Lambda_{m}}\right)=\frac{\beta_{m f} \beta_{f m}}{\left(\delta_{m}+\mu_{m}\right)\left(\delta_{f}+\mu_{f}\right)}(1-\tau)\left(1-\tau \frac{v-\Lambda_{f}}{\Lambda_{m}}\right), & v>\Lambda_{f} .\end{cases}
$$

(ii) When $\Lambda_{f}>\Lambda_{m}$

$$
h(v)= \begin{cases}R_{0}^{2}\left(0, \frac{v}{\Lambda_{m}}\right)=\frac{\beta_{m f} \beta_{f m}}{\left(\delta_{m}+\mu_{m}\right)\left(\delta_{f}+\mu_{f}\right)}\left(1-\tau \frac{v}{\Lambda_{m}}\right), & v \leq \Lambda_{m}, \\ R_{0}^{2}\left(\frac{v-\Lambda_{m}}{\Lambda_{f}}, 1\right)=\frac{\beta_{m f} \beta_{f m}}{\left(\delta_{m}+\mu_{m}\right)\left(\delta_{f}+\mu_{f}\right)}\left(1-\tau \frac{v-\Lambda_{m}}{\Lambda_{f}}\right)(1-\tau), & v>\Lambda_{m} .\end{cases}
$$


For Case (i), the derivative is

$$
h^{\prime}(v)= \begin{cases}-\frac{\beta_{m f} \beta_{f m}}{\left(\delta_{m}+\mu_{m}\right)\left(\delta_{f}+\mu_{f}\right)} \frac{\tau}{\Lambda_{f}}, & v \leq \Lambda_{f}, \\ -\frac{\beta_{m f} \beta_{f m}}{\left(\delta_{m}+\mu_{m}\right)\left(\delta_{f}+\mu_{f}\right)}(1-\tau) \frac{\tau}{\Lambda_{m}}, & v>\Lambda_{f} .\end{cases}
$$

We have $h^{\prime}(v)<0$. Since $\Lambda_{f} \leq \Lambda_{m}$, we have $\frac{1}{\Lambda_{f}}>\frac{1-\tau}{\Lambda_{m}}$. Therefore, $\left|h^{\prime}(v)\right|$ is smaller for $v \leq \Lambda_{f}$ than that for $v>\Lambda_{f}$. This shows that $h(v)$ is a decreasing function and the decline speed when $v \leq \Lambda_{f}$ is greater than that when $v>\Lambda_{f}$. This means that the vaccination becomes less effective once vaccine shots exceed the number of female recruits. A similar conclusion can be drawn for Case (ii).

\section{Appendix H - Estimation of the numbers of 14-year-old boys and girls in Guangxi in 2021- 2033}

Under-five mortality rate (U5MR) is the probability of dying by age 5 per 1000 live births. The probability of dying among children aged 5-14 is about $18 \%$ of U5MR in the same year $[62,63]$. Therefore, we estimate the number of children who are alive by age 14 to be newborn* $(1-$ U5MR-U5MR*18\%). The national census is only conducted at the year ending with 0 , and the $1 \%$ national sample census is conducted at the year ending with 5. According to [64, 65], the sex ratio (male/female) is 115.6 (assume females are 100) for newborns in 1995 in China, and it is 112.06 for 15 -year-old children in 2010. Thus, we estimate the sex ratio at age 14 to be the sex ratio at newborn (same cohort) $-\frac{14}{15} \times(115.6-112.06)$. Using the number of 14 year-old children and the sex ratio for age 14, we can estimate the numbers of 14-year-old girls and boys in 2021-2033. The results are given in Table 8.

\section{Acknowledgments}

This work was supported by the NSF grants DMS-1951595 (MM), DMS-1620957 (MH) and DMS-1950254 (LR).

\section{References}

[1] WHO, HPV: key facts, https://www.who.int/news-room/fact-sheets/detail/humanpapillomavirus-(hpv)-and-cervical-cancer (2021).

[2] J. Lei, A. Ploner, K. M. Elfström, J. Wang, A. Roth, F. Fang, K. Sundström, J. Dillner, P. Sparén, HPV vaccination and the risk of invasive cervical cancer, New England Journal of Medicine 383 (14) (2020) 1340-1348. 
[3] E. Petrosky, J. A. Bocchini Jr, S. Hariri, H. Chesson, C. R. Curtis, M. Saraiya, E. R. Unger, L. E. Markowitz, Use of 9-valent human papillomavirus (HPV) vaccine: updated HPV vaccination recommendations of the advisory committee on immunization practices, Morbidity and Mortality Weekly Report 64 (11) (2015) 300.

[4] WHO, HPV: News, https://www.who.int/news-room/detail/31-10-2019-major-milestonereached-as-100-countries-have-introduced-hpv-vaccine-into-national-schedule (2019).

[5] Australian Government Department of Health, Human papillomavirus (HPV) immunisation service, https://www.health.gov.au/health-topics/immunisation/immunisationservices/human-papillomavirus-hpv-immunisation-service (2021).

[6] Centers for Disease Control and Prevention (CDC), Human Papillomavirus (HPV) Fact Sheet, https://www.cdc.gov/std/hpv/stdfact-hpv.htm (2021).

[7] A. Green, HPV vaccine to be offered to boys in England, The Lancet 392 (10145) (2018) 374.

[8] S. Arie, HPV: WHO calls for countries to suspend vaccination of boys, BMJ: British Medical Journal (Online) 367.

URL https: //www . bmj . com/content/367/bmj . 16765

[9] M. Stanley, Perspective: vaccinate boys too, Nature 488 (7413) (2012) S10-S10.

[10] M. Brisson, É. Bénard, M. Drolet, J. A. Bogaards, I. Baussano, S. Vänskä, M. Jit, M.-C. Boily, M. A. Smith, J. Berkhof, et al., Population-level impact, herd immunity, and elimination after human papillomavirus vaccination: a systematic review and meta-analysis of predictions from transmission-dynamic models, The Lancet Public Health 1 (1) (2016) e8-e17.

[11] E. H. Elbasha, E. J. Dasbach, R. P. Insinga, Model for assessing human papillomavirus vaccination strategies, Emerging Infectious Diseases 13 (1) (2007) 28.

[12] E. H. Elbasha, E. J. Dasbach, R. P. Insinga, A multi-type HPV transmission model, Bulletin of Mathematical Biology 70 (8) (2008) 2126-2176.

[13] E. H. Elbasha, E. J. Dasbach, Impact of vaccinating boys and men against HPV in the United States, Vaccine 28 (42) (2010) 6858-6867.

[14] R. P. Insinga, E. J. Dasbach, E. H. Elbasha, A. Puig, L. M. Reynales-Shigematsu, Costeffectiveness of quadrivalent human papillomavirus (HPV) vaccination in Mexico: a transmission dynamic model-based evaluation, Vaccine 26 (1) (2007) 128-139.

[15] F. S. Mennini, P. Bonanni, F. Bianic, C. de Waure, G. Baio, G. Plazzotta, M. Uhart, A. Rinaldi, N. Largeron, Cost-effectiveness analysis of the nine-valent HPV vaccine in Italy, Cost Effectiveness and Resource Allocation 15 (1) (2017) 1-14. 
[16] P. Cody, K. Tobe, M. Abe, E. H. Elbasha, Public health impact and cost effectiveness of routine and catch-up vaccination of girls and women with a nine-valent HPV vaccine in Japan: a model-based study, BMC Infectious Diseases 21 (1) (2021) 1-13.

[17] L. Majed, X. Bresse, N. El Mouaddin, A. Schmidt, V. J. Daniels, A. Pavelyev, L. LevyBachelot, E. Elbasha, Public health impact and cost-effectiveness of a nine-valent genderneutral HPV vaccination program in France, Vaccine 39 (2) (2021) 438-446.

[18] V. Daniels, V. S. Prabhu, C. Palmer, S. Samant, S. Kothari, C. Roberts, E. Elbasha, Public health impact and cost-effectiveness of catch-up 9-valent HPV vaccination of individuals through age 45 years in the United States, Human Vaccines \& Immunotherapeutics (2021) $1-9$.

[19] H.-L. Peng, S. Tam, L. Xu, K. R. Dahlstrom, C.-F. Wu, S. Fu, C. Zhong, W. Chan, E. M. Sturgis, L. Ramondetta, et al., Age-structured population modeling of HPV-related cervical cancer in Texas and US, Scientific Reports 8 (1) (2018) 1-12.

[20] C. Zhong, L. Xu, H.-L. Peng, S. Tam, K. R. Dahlstrom, C.-F. Wu, S. Fu, W. Chan, E. M. Sturgis, L. M. Ramondetta, et al., An economic and disease transmission model of human papillomavirus and oropharyngeal cancer in Texas, Scientific Reports 11 (1) (2021) 1-16.

[21] K. Haeussler, A. Marcellusi, F. S. Mennini, G. Favato, M. Picardo, G. Garganese, M. Bononi, S. Costa, G. Scambia, P. Zweifel, et al., Cost-effectiveness analysis of universal human papillomavirus vaccination using a dynamic Bayesian methodology: the BEST II study, Value in Health 18 (8) (2015) 956-968.

[22] J. Kim, B. Andres-Beck, S. Goldie, The value of including boys in an HPV vaccination programme: a cost-effectiveness analysis in a low-resource setting, British Journal of Cancer 97 (9) (2007) 1322-1328.

[23] H. W. Chesson, D. U. Ekwueme, M. Saraiya, E. F. Dunne, L. E. Markowitz, The costeffectiveness of male HPV vaccination in the United States, Vaccine 29 (46) (2011) 84438450.

[24] J. J. Kim, S. J. Goldie, Health and economic implications of HPV vaccination in the United States, New England Journal of Medicine 359 (8) (2008) 821-832.

[25] F. Saldaña, J. A. Camacho-Gutíerrez, I. Barradas, A. Korobeinikov, When optimal is not the best: cost effectiveness analysis for HPV epidemic models, arXiv preprint arXiv:2011.06594.

[26] O. Damm, J. Horn, R. T. Mikolajczyk, M. E. Kretzschmar, A. M. Kaufmann, Y. Deleré, B. Ultsch, O. Wichmann, A. Krämer, W. Greiner, Cost-effectiveness of human papillomavirus vaccination in Germany, Cost Effectiveness and Resource Allocation 15 (1) (2017) 1-19. 
[27] S. Gao, M. Martcheva, H. Miao, L. Rong, A dynamic model to assess human papillomavirus vaccination strategies in a heterosexual population combined with men who have sex with men, Bulletin of Mathematical Biology 83 (1) (2021) 1-36.

[28] J. A. Bogaards, M. Kretzschmar, M. Xiridou, C. J. Meijer, J. Berkhof, J. Wallinga, Sexspecific immunization for sexually transmitted infections such as human papillomavirus: insights from mathematical models, PLoS Med 8 (12) (2011) e1001147.

[29] R. V. Barnabas, G. P. Garnett, 5. The potential public health impact of vaccines against human papillomavirus, HPV Handbook (2004) 61.

[30] M. Brisson, N. van de Velde, E. L. Franco, M. Drolet, M.-C. Boily, Incremental impact of adding boys to current human papillomavirus vaccination programs: role of herd immunity, Journal of Infectious Diseases 204 (3) (2011) 372-376.

[31] R. V. Barnabas, P. Laukkanen, P. Koskela, O. Kontula, M. Lehtinen, G. P. Garnett, Epidemiology of HPV 16 and cervical cancer in Finland and the potential impact of vaccination: mathematical modelling analyses, PLoS Med 3 (5) (2006) e138.

[32] F. Azevedo, L. Esteva, C. P. Ferreira, Assessing the impact of prophylactic vaccines on HPV prevalence, TEMA (São Carlos) 20 (2) (2019) 305-321.

[33] J. Horn, O. Damm, M. Kretzschmar, Y. Deleré, O. Wichmann, A. Kaufmann, E. Garbe, A. Krämer, W. Greiner, R. Mikolajczyk, Estimating the long-term effects of HPV vaccination in Germany, Vaccine 31 (19) (2013) 2372-2380.

[34] V. Brown, K. A. White, The HPV vaccination strategy: could male vaccination have a significant impact?, Computational and Mathematical Methods in Medicine 11 (3) (2010) 223-237.

[35] E. H. Elbasha, Impact of prophylactic vaccination against human papillomavirus infection, Contemporary Mathematics 410 (2006) 113-128.

[36] E. H. Elbasha, Global stability of equilibria in a two-sex HPV vaccination model, Bulletin of Mathematical Biology 70 (3) (2008) 894.

[37] L. Ribassin-Majed, R. Lounes, S. Clémençon, Deterministic modelling for transmission of Human Papillomavirus 6/11: impact of vaccination, Mathematical Medicine and Biology: a Journal of the IMA 31 (2) (2014) 125-149.

[38] N. Ziyadi, A male-female mathematical model of human papillomavirus (HPV) in African American population, Mathematical Biosciences \& Engineering 14 (1) (2017) 339.

[39] O. Sharomi, T. Malik, A model to assess the effect of vaccine compliance on Human Papillomavirus infection and cervical cancer, Applied Mathematical Modelling 47 (2017) 528-550. 
[40] K. Zhang, X. W. Wang, H. Liu, Y. P. Ji, Q. Pan, Y. M. Wei, X. Ma, Mathematical analysis of a human papillomavirus transmission model with vaccination and screening, Mathematical Biosciences and Engineering 17 (5) (2020) 5449-5476.

[41] A. Omame, R. Umana, D. Okuonghae, S. Inyama, Mathematical analysis of a two-sex Human Papillomavirus (HPV) model, International Journal of Biomathematics 11 (07) (2018) 1850092.

[42] T. Malik, A. Gumel, E. H. Elbasha, Qualitative analysis of an age-and sex-structured vaccination model for human papillomavirus, Discrete \& Continuous Dynamical Systems-B 18 (8) (2013) 2151.

[43] T. Malik, J. Reimer, A. Gumel, E. H. Elbasha, S. Mahmud, The impact of an imperfect vaccine and pap cytology screening on the transmission of human papillomavirus and occurrence of associated cervical dysplasia and cancer, Mathematical Biosciences \& Engineering 10 (4) (2013) 1173.

[44] C. Muñoz-Quiles, J. Díez-Domingo, L. Acedo, V. Sánchez-Alonso, R. J. Villanueva, On the elimination of infections related to oncogenic human papillomavirus: An approach using a computational network model, Viruses 13 (5) (2021) 906.

[45] L. Acedo, J.-C. Cortés, J. Díez-Domingo, V. Sánchez-Alonso, J. Tuells, R.-J. Villanueva, Uncertainty and sensitivity of the sexual behavior changes to the current human papillomavirus vaccination campaign in spain, Mathematical Methods in the Applied Sciences 44 (9) (2021) $7845-7857$.

[46] J. Díez-Domingo, V. Sánchez-Alonso, R.-J. Villanueva, L. Acedo, J. Tuells, Impact of a gender-neutral HPV vaccination program in men who have sex with men (MSM), International Journal of Environmental Research and Public Health 18 (3) (2021) 963.

[47] S. L. Ranjeva, E. B. Baskerville, V. Dukic, L. L. Villa, E. Lazcano-Ponce, A. R. Giuliano, G. Dwyer, S. Cobey, Recurring infection with ecologically distinct HPV types can explain high prevalence and diversity, Proceedings of the National Academy of Sciences 114 (51) (2017) 13573-13578.

[48] H. Trottier, S. Ferreira, P. Thomann, M. C. Costa, J. S. Sobrinho, J. C. M. Prado, T. E. Rohan, L. L. Villa, E. L. Franco, Human papillomavirus infection and reinfection in adult women: the role of sexual activity and natural immunity, Cancer Research 70 (21) (2010) 8569-8577.

[49] P. Van den Driessche, J. Watmough, Reproduction numbers and sub-threshold endemic equilibria for compartmental models of disease transmission, Mathematical Biosciences 180 (1-2) (2002) 29-48. 
[50] F. Wei, M. Guo, S. Huang, M. Li, X. Cui, Y. Su, X. Wu, X. Ma, Y. Zheng, Y. Huang, et al., Sex differences in the incidence and clearance of anogenital human papillomavirus infection in Liuzhou, China: an observational cohort study, Clinical Infectious Diseases 70 (1) (2020) 82-89.

[51] F. Wei, K. Yin, X. Wu, J. Lan, S. Huang, W. Sheng, J. Zhao, Y. Su, Y. Wang, Y. Li, et al., Human papillomavirus prevalence and associated factors in women and men in south China: a population-based study, Emerging Microbes \& Infections 5 (1) (2016) 1-8.

[52] Liuzhou statistical yearbook, http://lztj.liuzhou.gov.cn/xxgk/jcxxgk/sjfb/tjnj/201811/ P020181113530838124483.pdf (2015).

[53] P. J. Woestenberg, A. J. King, B. H. Van Benthem, R. Donken, S. Leussink, F. R. Van Der Klis, H. E. De Melker, M. A. Van Der Sande, C. J. Hoebe, J. A. Bogaards, et al., Bivalent vaccine effectiveness against type-specific HPV positivity: evidence for cross-protection against oncogenic types among Dutch STI clinic visitors, The Journal of Infectious Diseases 217 (2) (2018) 213-222.

[54] S. Marino, I. B. Hogue, C. J. Ray, D. E. Kirschner, A methodology for performing global uncertainty and sensitivity analysis in systems biology, Journal of Theoretical Biology 254 (1) (2008) 178-196.

[55] R. Taylor, Interpretation of the correlation coefficient: a basic review, Journal of Diagnostic Medical Sonography 6 (1) (1990) 35-39.

[56] M. Riesen, V. Garcia, N. Low, C. L. Althaus, Modeling the consequences of regional heterogeneity in human papillomavirus (HPV) vaccination uptake on transmission in Switzerland, Vaccine 35 (52) (2017) 7312-7321.

[57] S. L. Lee, A. M. Tameru, A mathematical model of human papillomavirus (HPV) in the United States and its impact on cervical cancer, Journal of Cancer 3 (2012) 262-268.

[58] A. F. Brouwer, M. C. Eisenberg, R. Meza, Age effects and temporal trends in HPV-related and HPV-unrelated oral cancer in the United States: a multistage carcinogenesis modeling analysis, PloS One 11 (3) (2016) e0151098.

[59] I. Baussano, G. Ronco, N. Segnan, K. French, P. Vineis, G. P. Garnett, HPV-16 infection and cervical cancer: modeling the influence of duration of infection and precancerous lesions, Epidemics 2 (1) (2010) 21-28.

[60] F. R. Gantmakher, The theory of matrices, Vol. 131, American Mathematical Soc., 1959.

[61] M. Martcheva, An introduction to mathematical epidemiology, Vol. 61, Springer, 2015. 
[62] Under-five mortality rate (probability of dying by age 5 per 1000 live births), https://www.who.int/data/gho/data/indicators/indicator-details/GHO/under-five-mortalityrate-(probability-of-dying-by-age-5-per-1000-live-births) (2021).

[63] Mortality among children aged 5-14 years, https://www.who.int/news-room/factsheets/detail/mortality-among-children-aged-5-14-years (2019).

[64] Figure 1.9 Sex ratio at birth, 19822017, https://www.unicef.cn/en/figure-19-sex-ratio-birth19822017 (2018).

[65] Tabulation on the 2010 Population Census of China, http://www.stats.gov.cn/tjsj/pcsj/rkpc/6rp/indexch.htm (2011).

[66] P. J. Woestenberg, A. J. King, M. A. van der Sande, R. Donken, S. Leussink, F. R. van der Klis, C. J. Hoebe, J. A. Bogaards, B. H. van Benthem, D. Adema, et al., No evidence for cross-protection of the HPV-16/18 vaccine against HPV-6/11 positivity in female STI clinic visitors, Journal of Infection 74 (4) (2017) 393-400. 
medRxiv preprint doi: https://doi.org/10.1101/2021.12.19.21268067; this version posted December 21, 2021. The copyright holder for this preprint (which was not certified by peer review) is the author/funder, who has granted medRxiv a license to display the preprint in perpetuity.

It is made available under a CC-BY-ND 4.0 International license .

Table 1: Description of variables and parameters.

\begin{tabular}{|c|c|c|c|c|c|}
\hline Symbol & Description & Baseline & Unit & Range & Source \\
\hline \multicolumn{6}{|l|}{ Subscripts } \\
\hline$f$ & Female & & & & \\
\hline$m$ & Male & & & & \\
\hline$k$ & Gender $(k=f, m)$ & & & & \\
\hline \multicolumn{6}{|l|}{ Variables } \\
\hline$S_{k}(t)$ & Susceptible population of gender $k$ & & & & \\
\hline$V_{k}(t)$ & Vaccinated population of gender $k$ & & & & \\
\hline$I_{k}(t)$ & Infected population of gender $k$ & & & & \\
\hline$N_{k}(t)$ & Total size of population of gender $k$ & & & & \\
\hline$N(t)$ & Total size of population & & & & \\
\hline$\lambda_{k}$ & Force of infection for gender $k$ & & & & \\
\hline \multicolumn{6}{|l|}{ Parameters } \\
\hline$\Lambda_{f}$ & Recruits into sexually active females & 340998 & person/year & {$[317422,391244]$} & See text \\
\hline$\Lambda_{m}$ & Recruits into sexually active males & 387463 & person/year & {$[342579,428756]$} & See text \\
\hline$\mu_{f}\left(\mu_{m}\right)$ & Exit rate from sexually active females (males) & $1 /(55-15)$ & $1 /$ year & See text & \\
\hline$\beta_{m f}$ & Transmission rate from males to females & Calibration & $1 /$ year & Calibration & See text \\
\hline$\beta_{f m}$ & Transmission rate from females to males & Calibration & $1 /$ year & Calibration & See text \\
\hline$\delta_{f}$ & Recovery rate from infection for females & $12 / 12.3$ & $1 /$ year & {$[12 / 13.3,12 / 7.7]$} & See text \\
\hline$\delta_{m}$ & Recovery rate from infection for males & $12 / 6.5$ & $1 /$ year & {$[12 / 7.7,12 / 6.2]$} & See text \\
\hline$\tau$ & Degree of protection by vaccine & 0.899 & none & {$[0.817,0.944]$} & {$[53]$} \\
\hline$\phi_{k}$ & Percentage of new recruits vaccinated for gender $k$ & varied & none & & \\
\hline$v$ & Amount of vaccines per year $\left(v=\sum_{k} \phi_{k} \Lambda_{k}\right)$ & varied & person/year & & \\
\hline dummy & Parameter for comparison in the PRCC & 1 & none & {$[1,10]$} & See text \\
\hline
\end{tabular}

Table 2: Calibration for $\beta_{m f}$ and $\beta_{f m}$ and the corresponding $R_{0}$ for different HPV types in people aged 15-55 in Guangxi.

\begin{tabular}{|l|c|c|c|c|c|c|c|}
\hline HPV types & $p_{f}$ & $p_{m}$ & $\delta_{f}$ & $\delta_{m}$ & $\beta_{m f}$ & $\beta_{f m}$ & $R_{0}$ \\
\hline HPV 16/18 & $107 / 2344$ & $30 / 1937$ & $12 / 12.3$ & $12 / 6.5$ & 2.8693 & 0.6967 & 1.0333 \\
HPV 6/11 & $32 / 2344$ & $23 / 1937$ & $12 / 6.7$ & $12 / 6.4$ & 1.9637 & 1.8068 & 1.0140 \\
HPV 31 & $0.8 \%$ & $0.2 \%$ & $12 / 6.5$ & $12 / 6.9$ & 6.999 & 0.4775 & 1.0062 \\
HPV 33 & $0.9 \%$ & $0.4 \%$ & $12 / 11.7$ & $12 / 6.1$ & 2.2147 & 0.9603 & 1.008 \\
HPV 45 & $0.4 \%$ & $0.5 \%$ & $12 / 6.7$ & $12 / 6.7$ & 1.3532 & 2.4648 & 1.0056 \\
HPV 52 & $6.6 \%$ & $1.5 \%$ & $12 / 13.1$ & $12 / 6.9$ & 4.1167 & 0.4398 & 1.0443 \\
HPV 58 & $3.5 \%$ & $3.5 \%$ & $12 / 12.7$ & $12 / 6.4$ & 0.9333 & 2.1271 & 1.0379 \\
\hline
\end{tabular}


medRxiv preprint doi: https://doi.org/10.1101/2021.12.19.21268067; this version posted December 21, 2021. The copyright holder for this preprint (which was not certified by peer review) is the author/funder, who has granted medRxiv a license to display the preprint in perpetuity.

It is made available under a CC-BY-ND 4.0 International license .

Table 3: PRCCs for $R_{0}$ and prevalences in females, males and the total population (* means significant).

\begin{tabular}{|c|c|c|c|c|}
\hline Inputs & $\operatorname{PRCC}\left(R_{0}\right)$ & $\operatorname{PRCC}\left(\frac{I_{f}}{N_{f}}\right)$ & $\operatorname{PRCC}\left(\frac{I_{m}}{N_{m}}\right)$ & $\operatorname{PRCC}\left(\frac{I_{f}+I_{m}}{N}\right)$ \\
\hline dummy & -0.0090 & -0.0060 & -0.0061 & -0.0068 \\
\hline$\phi_{m}$ & $-0.9244^{*}$ & $-0.9004^{*}$ & $-0.9043^{*}$ & $-0.9027^{*}$ \\
\hline$\phi_{f}$ & $-0.9241^{*}$ & $-0.9042^{*}$ & $-0.8975^{*}$ & $-0.9038^{*}$ \\
\hline$\tau$ & -0.4139 & -0.3508 & -0.3481 & -0.3528 \\
\hline$\delta_{m}$ & -0.2695 & $-0.7337^{*}$ & $-0.7369^{*}$ & $-0.7376^{*}$ \\
\hline$\delta_{f}$ & $-0.5623^{*}$ & $-0.9486^{*}$ & $-0.9454^{*}$ & $-0.9486^{*}$ \\
\hline$\beta_{f m}$ & 0.2712 & $0.6596^{*}$ & $0.6619^{*}$ & $0.6634^{*}$ \\
\hline$\beta_{m f}$ & $0.5599^{*}$ & $0.9190^{*}$ & $0.9144^{*}$ & $0.9191^{*}$ \\
\hline$\mu$ & 0.0010 & 0.0139 & 0.0130 & $0.0133^{*}$ \\
\hline$\Lambda_{f}$ & & -0.1156 & -0.1425 & -0.1297 \\
\hline$\Lambda_{m}$ & & -0.1308 & -0.1027 & -0.1182 \\
\hline
\end{tabular}

Table 4: Time and total vaccines needed to reduce the prevalence in the total population to below 0.0005 given fixed vaccine amount every year. All vaccines are given to females. The following vaccine coverage refers to the coverage of 15-year-old girls per year.

\begin{tabular}{|c|c|c|c|}
\hline Vaccines (per year) & Vaccine coverage & Time (year) & Total vaccines \\
\hline 50000 & $\phi_{f}=0.15$ & $0-127.3598$ & 6367990 \\
60000 & $\phi_{f}=0.18$ & $0-103.4169$ & 6205014 \\
70000 & $\phi_{f}=0.21$ & $0-88.6719$ & 6207033 \\
80000 & $\phi_{f}=0.23$ & $0-78.2606$ & 6260848 \\
90000 & $\phi_{f}=0.26$ & $0-70.6311$ & 6356799 \\
100000 & $\phi_{f}=0.29$ & $0-64.6396$ & 6463960 \\
110000 & $\phi_{f}=0.32$ & $0-59.8739$ & 6586129 \\
120000 & $\phi_{f}=0.35$ & $0-55.9049$ & 6708588 \\
130000 & $\phi_{f}=0.38$ & $0-52.5262$ & 6828460 \\
140000 & $\phi_{f}=0.41$ & $0-49.8603$ & 6980442 \\
150000 & $\phi_{f}=0.44$ & $0-47.3095$ & 7096425 \\
\hline
\end{tabular}


medRxiv preprint doi: https://doi.org/10.1101/2021.12.19.21268067; this version posted December 21, 2021. The copyright holder for this preprint (which was not certified by peer review) is the author/funder, who has granted medRxiv a license to display the preprint in perpetuity.

It is made available under a CC-BY-ND 4.0 International license.

Table 5: Time and total vaccines needed to reduce the prevalence in the total population to below 0.0005 given different vaccination strategies. All vaccines are given to females. The following vaccine coverage refers to the coverage of 15 -year-old girls per year.

\begin{tabular}{|c|c|c|c|}
\hline Vaccines (per year) & Vaccine coverage & Time (year) & Total vaccines \\
\hline 60000 & $\phi_{f}=0.18$ & $0-103.4169$ & 6205014 \\
\hline 80000 & $\phi_{f}=0.23$ & $0-50$ & 5711221 \\
40000 & $\phi_{f}=0.12$ & $51-92.78052$ & \\
\hline 100000 & $\phi_{f}=0.29$ & $0-30$ & \\
65000 & $\phi_{f}=0.19$ & $31-60$ & 5555080 \\
30000 & $\phi_{f}=0.09$ & $61-80.16933$ & \\
\hline 120000 & $\phi_{f}=0.35$ & $0-20$ & \\
90000 & $\phi_{f}=0.26$ & $21-40$ & 5663935 \\
60000 & $\phi_{f}=0.18$ & $41-60$ & \\
30000 & $\phi_{f}=0.09$ & $61-68.79783$ & \\
\hline
\end{tabular}

Table 6: Some vaccination strategies with total time less then 68 years and total vaccines less than 5.5 million in order to reduce the prevalence in the total population to below 0.0005 . All vaccines are given to females. The following vaccine coverage refers to the coverage of 15 -year-old girls per year. The integer $n=1,2, \cdots$ represents the number of period.

\begin{tabular}{|c|c|c|c|c|c|}
\hline $\begin{array}{c}\text { Period length } \\
\text { (year) }\end{array}$ & $\begin{array}{c}\text { Vaccines in the first } \\
\text { period (per year) }\end{array}$ & $\begin{array}{c}\text { Vaccine } \\
\text { deduction }\end{array}$ & vaccine coverage & $\begin{array}{c}\text { Total time } \\
\text { (year) }\end{array}$ & $\begin{array}{c}\text { Total } \\
\text { vaccines }\end{array}$ \\
\hline 10 & 150000 & 25000 & $\phi_{f}=0.44-0.07 \mathrm{n}$ & 67.125 & 5250000 \\
5 & 175000 & 15000 & $\phi_{f}=0.51-0.04 \mathrm{n}$ & 58.0625 & 5225000 \\
2 & 178000 & 6000 & $\phi_{f}=0.52-0.02 \mathrm{n}$ & 59.225 & 5382950 \\
\hline
\end{tabular}


medRxiv preprint doi: https://doi.org/10.1101/2021.12.19.21268067; this version posted December 21, 2021. The copyright holder for this preprint (which was not certified by peer review) is the author/funder, who has granted medRxiv a license to display the preprint in perpetuity.

It is made available under a CC-BY-ND 4.0 International license .

Table 7: The values of $R_{0}$ with different vaccine distributions.

\begin{tabular}{|c|c|c|}
\hline$\phi_{f}$ & $\phi_{m}$ & $R_{0}$ \\
\hline 0 & 0 & 1.0333 \\
\hline \multirow{3}{*}{0.25} & 0 & 0.9098 \\
\cline { 2 - 3 } & 0.125 & 0.8572 \\
\cline { 2 - 3 } & 0.25 & 0.8011 \\
\hline \multirow{4}{*}{0.5} & 0 & 0.7667 \\
\cline { 2 - 3 } & 0.25 & 0.675 \\
\cline { 2 - 3 } 0.75 & 0.5 & 0.5688 \\
\hline \multirow{3}{*}{0.375} & 0.4802 \\
\cline { 2 - 3 } & 0.75 & 0.3366 \\
\hline \multirow{3}{*}{1} & 0 & 0.3284 \\
\cline { 2 - 3 } & 0.5 & 0.2436 \\
\cline { 2 - 3 } & 1 & 0.1044 \\
\hline
\end{tabular}

Table 8: Estimation of the numbers of 14-year-old girls and boys in 2021-2033 from newborns in 2007-2019*.

\begin{tabular}{|cccc|ccccc|}
\hline Year & Newborn & U5MR & $\begin{array}{c}\text { Sex ratio } \\
\text { (newborn) }\end{array}$ & Year & $\begin{array}{c}\text { 14-year } \\
\text { children }\end{array}$ & $\begin{array}{c}\text { Sex ratio } \\
\text { (age 14) }\end{array}$ & $\begin{array}{c}\text { 14-year } \\
\text { girls }\end{array}$ & $\begin{array}{c}\text { 14-year } \\
\text { boys }\end{array}$ \\
\hline 2007 & 710000 & 15.77 & 120.68 & 2021 & 696787.9 & 117.376 & 326623 & 383377 \\
\hline 2008 & 720000 & 13.4 & 121.12 & 2022 & 708615.4 & 117.816 & 330554 & 389446 \\
\hline 2009 & 720000 & 11.22 & 121.56 & 2023 & 710467.5 & 118.256 & 329888 & 390112 \\
\hline 2010 & 720000 & 10.88 & 122 & 2024 & 710756.4 & 118.696 & 329224 & 390776 \\
\hline 2011 & 710000 & 9.7 & 120.4 & 2025 & 701873.3 & 117.096 & 327044 & 382956 \\
\hline 2012 & 740000 & 7.89 & 118.8 & 2026 & 733110.5 & 115.496 & 343394 & 396606 \\
\hline 2013 & 750000 & 7.73 & 117.2 & 2027 & 743159 & 113.896 & 350638 & 399362 \\
\hline 2014 & 720000 & 7.74 & 115.6 & 2028 & 713424.1 & 112.296 & 339149 & 380851 \\
\hline 2015 & 720000 & 6.25 & 114 & 2029 & 714690 & 110.696 & 341725 & 378275 \\
\hline 2016 & 770000 & 6.03 & 113.446 & 2030 & 764521.1 & 110.142 & 366419 & 403581 \\
\hline 2017 & 820000 & 4.99 & 112.892 & 2031 & 815171.7 & 109.588 & 391244 & 428756 \\
\hline 2018 & 710000 & 4.98 & 112.338 & 2032 & 705827.8 & 109.034 & 339658 & 370342 \\
\hline 2019 & 660000 & 4.77 & 111.23 & 2033 & 656285.1 & 107.926 & 317421 & 342579 \\
\hline
\end{tabular}

*U5MR: under 5 mortality rate; Sex ratio: male/female (female is 100); The number of 14-year children is derived from the number of newborn and U5MR; Sex ratio at age 14 is derived from sex ratio at newborn; Numbers of 14-years girls and boys are derived from the number of 14-year children and the sex ratio at age 14 (for details see Appendix H). 
medRxiv preprint doi: https://doi.org/10.1101/2021.12.19.21268067; this version posted December 21, 2021. The copyright holder for this preprint (which was not certified by peer review) is the author/funder, who has granted medRxiv a license to display the preprint in perpetuity.

It is made available under a CC-BY-ND 4.0 International license.

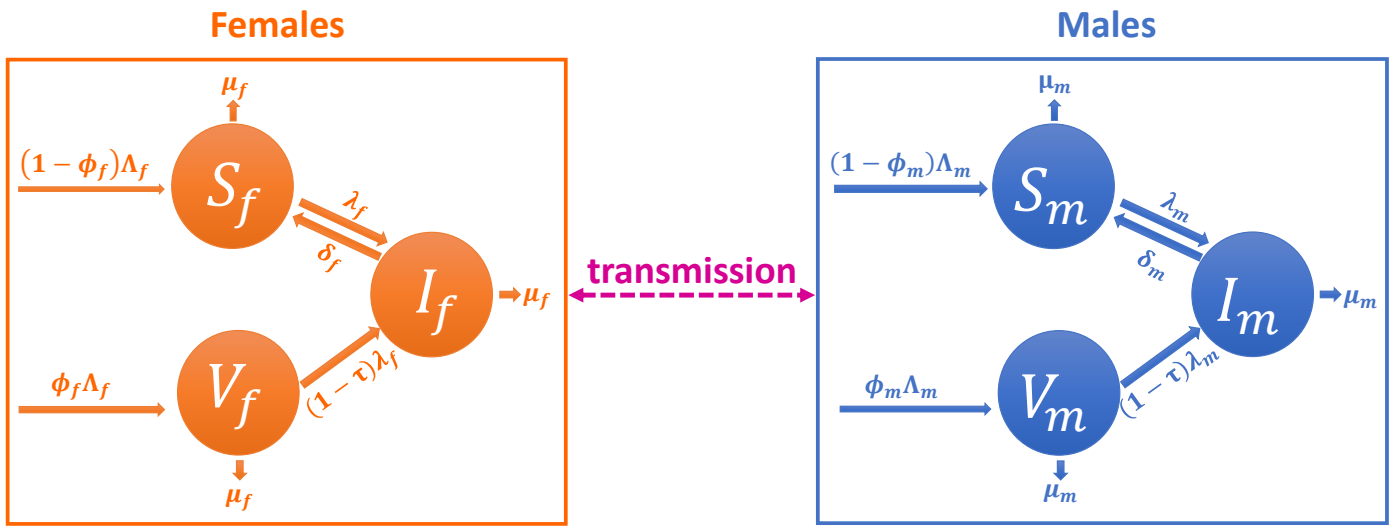

Figure 1: Flow diagram of the model of HPV infection with vaccination. Each group (females and males, denoted by $f$ and $m$, respectively) is divided into three subgroups: susceptible, infected and vaccinated, denoted by $S, I$ and $V$, respectively. The transmission happens between females and males. Descriptions of parameters are given in Table 1.

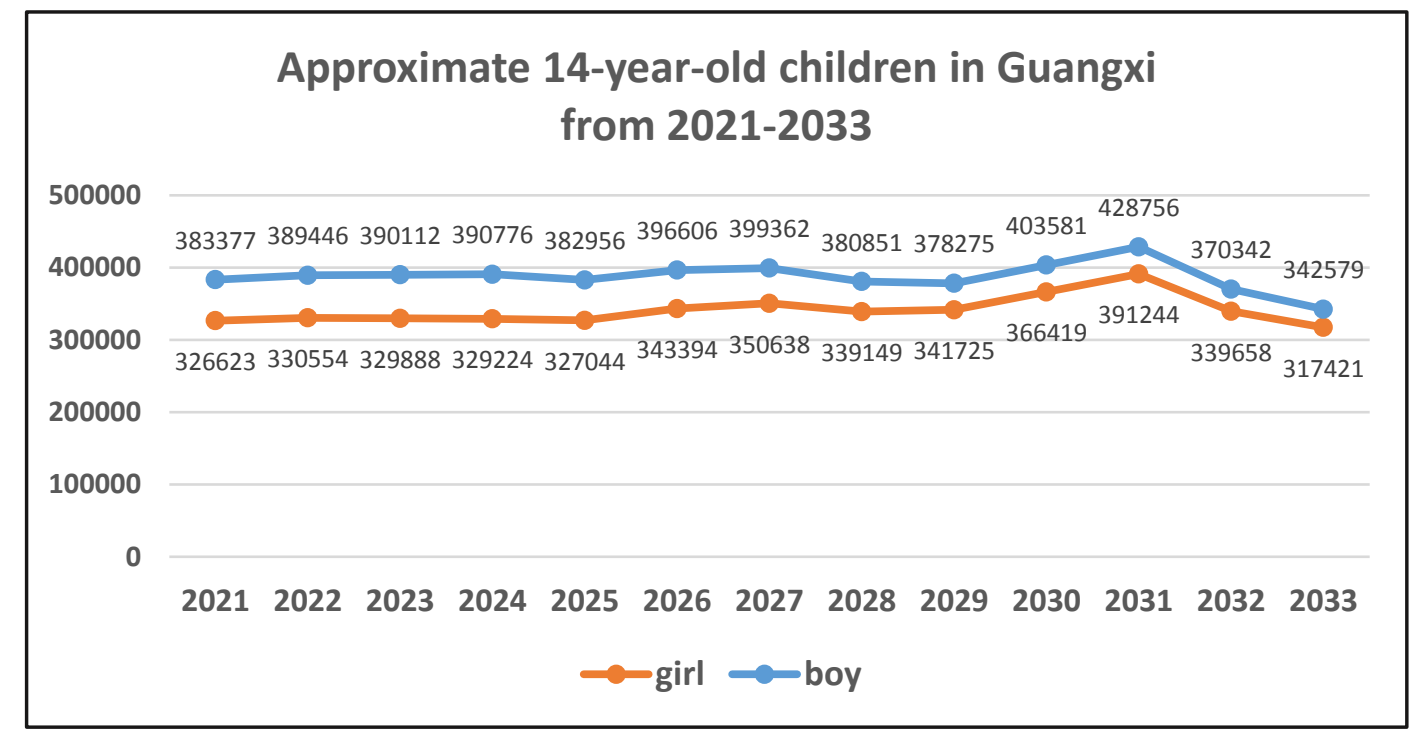

Figure 2: The recruits to susceptible for Guangxi Province. We use data from Liuzhou for parameter calibration and apply to Guangxi. We assume that 14-year-old children are vaccinated and estimate the target population size in 20212033 by newborns in 2007-2019. 
medRxiv preprint doi: https://doi.org/10.1101/2021.12.19.21268067; this version posted December 21, 2021. The copyright holder for this preprint (which was not certified by peer review) is the author/funder, who has granted medRxiv a license to display the preprint in perpetuity.

It is made available under a CC-BY-ND 4.0 International license.
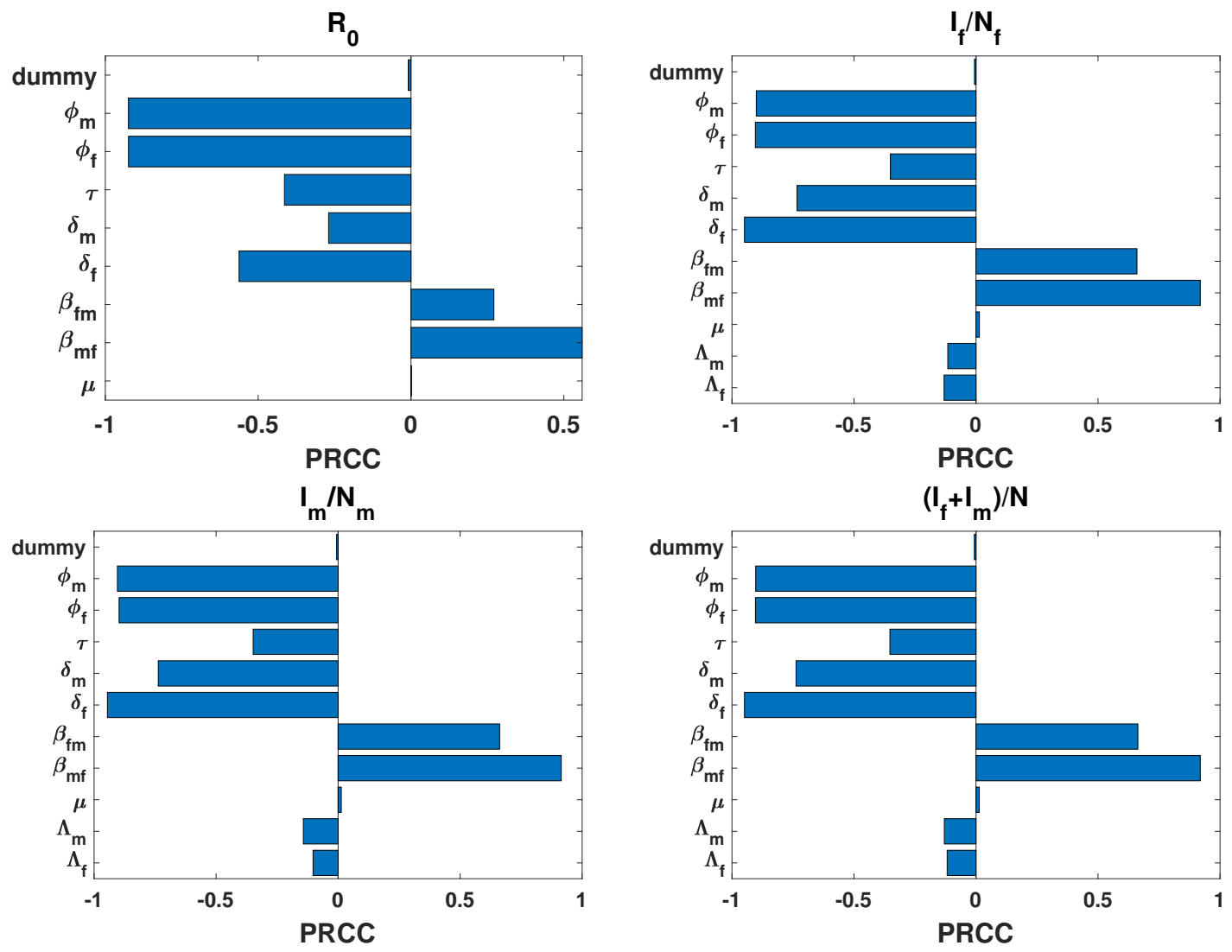

Figure 3: Sensitivity analysis using PRCC. In all panels, we choose $0.08,0.01$ as base values for $\phi_{f}$ and $\phi_{m}$, respectively, and $[0.01,0.99]$ as their range. We fix $\mu_{f}=\mu_{m}=\mu=1 /(55-15)$. The other parameters are from Table 1 . The initial conditions for (b)-(d) are the pre-vaccination endemic equilibria and the end of time is 50 years. 
(a)

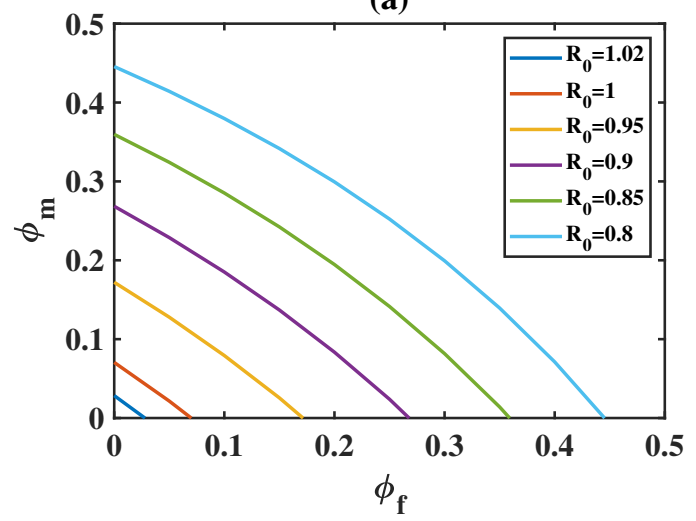

(c)

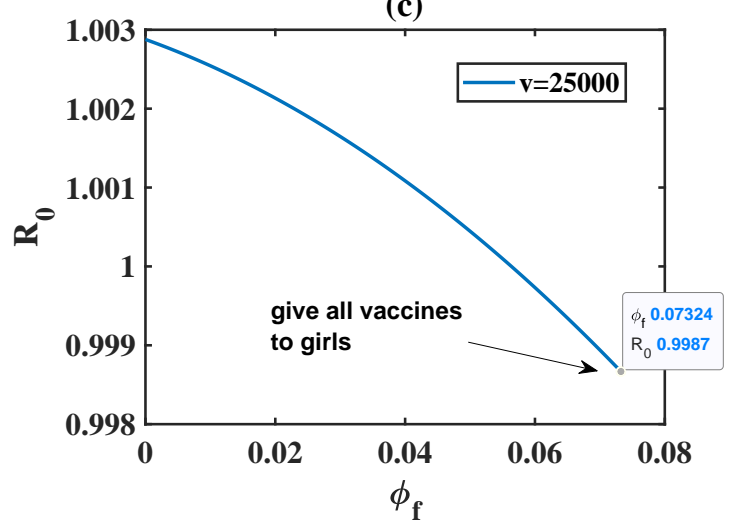

(e)

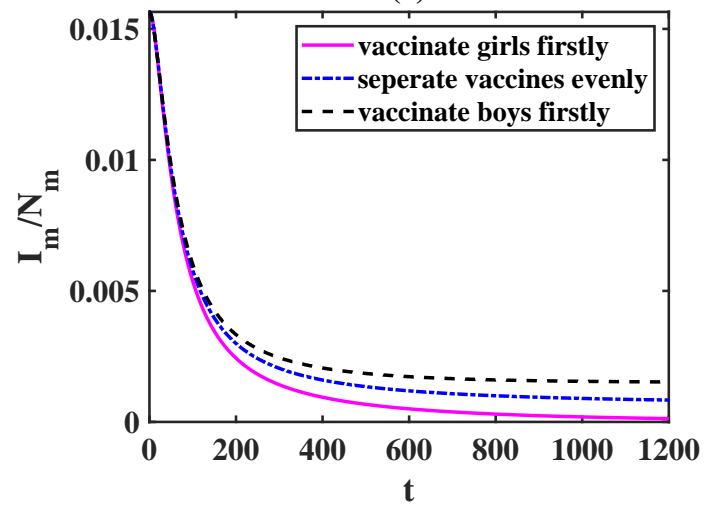

(b)

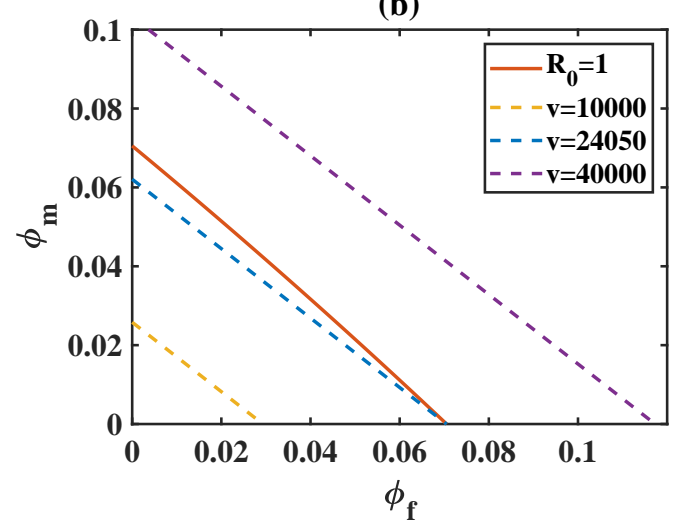

(d)

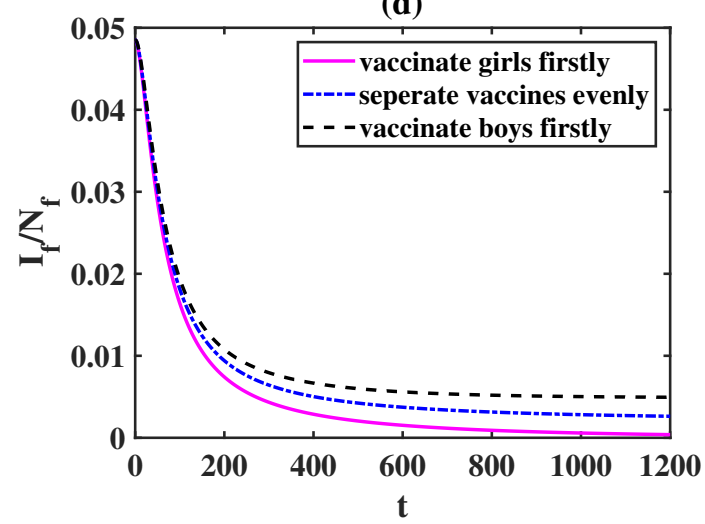

(f)

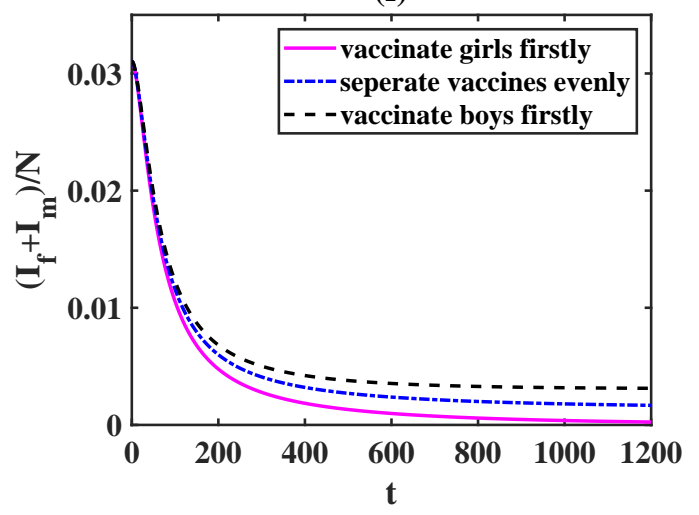

Figure 4: (a) The basic reproduction number $R_{0}$ with different vaccine distributions. (b) The minimum vaccine amount v needed per year to achieve $R_{0}=1$ using linear programming. (c) $R_{0}$ for $v=25000$. The vaccination proportion for males $\phi_{m}$ can be calculated according to the vaccine amount $v$ and vaccination proportion for females $\phi_{f}$. (d-f) Prevalences in females, males and the total population with different vaccine distributions for $v=25000$. The other parameters are from Table 1. The initial conditions for (d-f) are the pre-vaccination endemic equilibria. It shows minimum $R_{0}$ is attained when all vaccines are given to girls, which results in lower prevalences in females, males and the total population. 


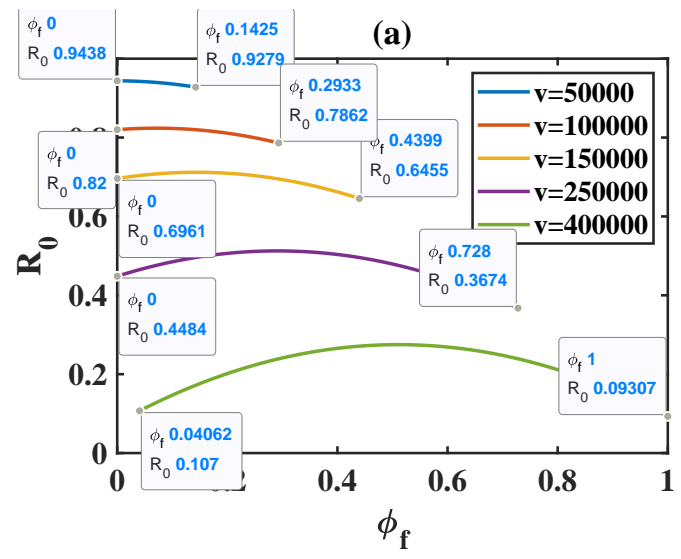

(c)

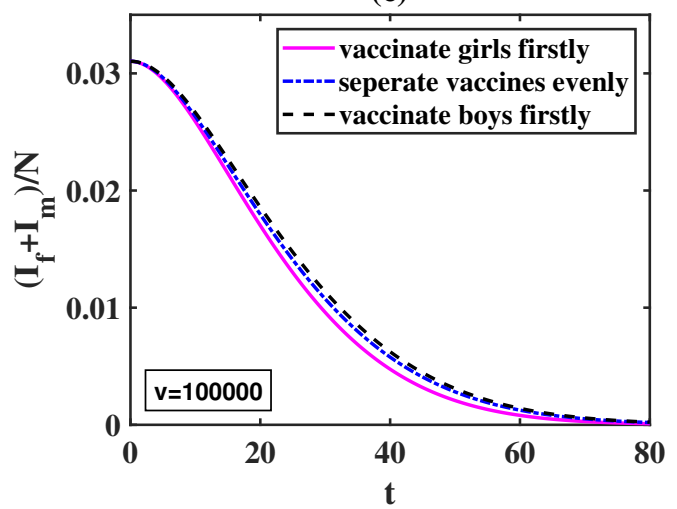

(e)

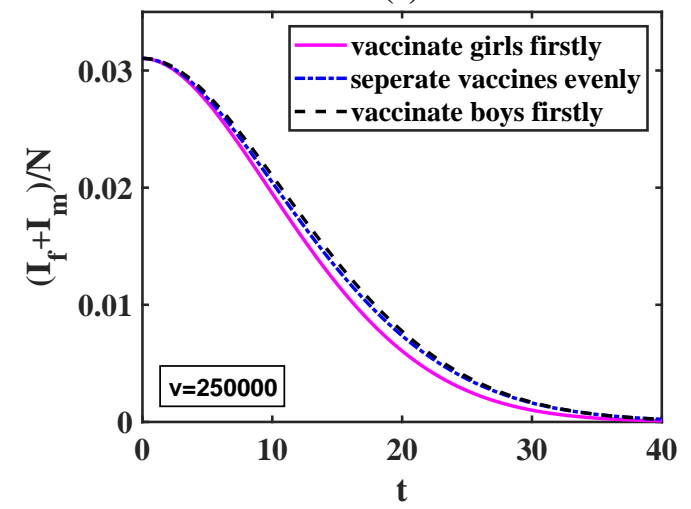

(b)

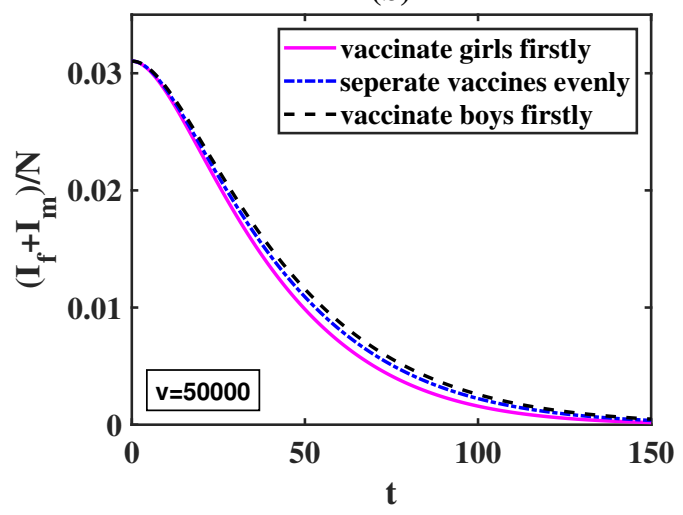

(d)

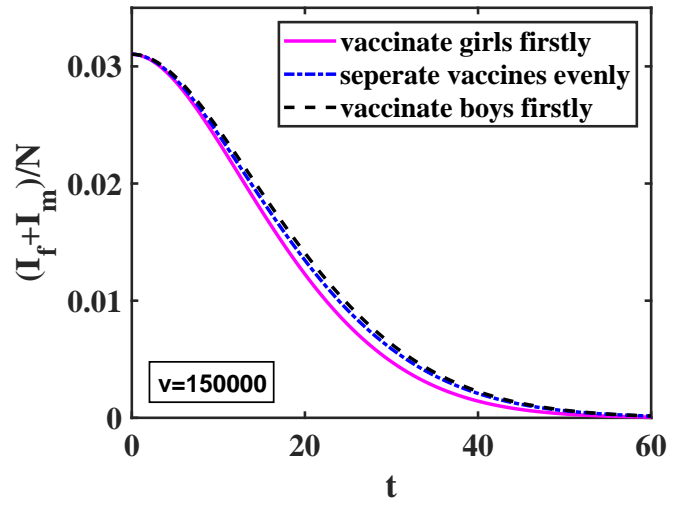

(f)

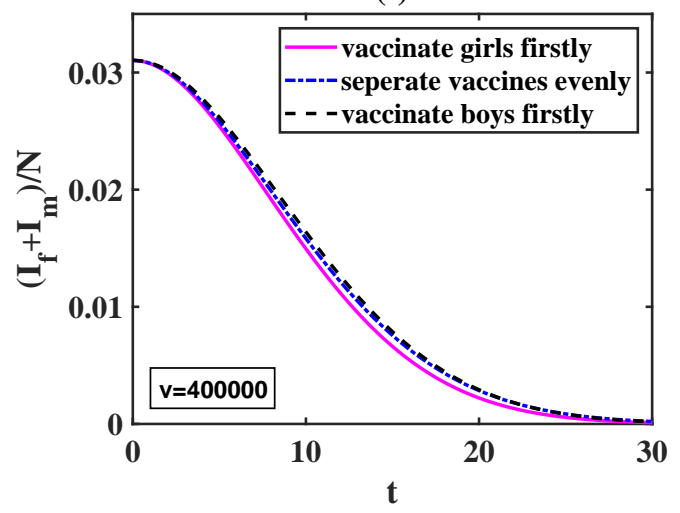

Figure 5: (a) The basic reproduction number $R_{0}$ for different vaccine amount $v$. The vaccination proportion for males $\phi_{m}$ can be calculated according to $v$ and the vaccination proportion for females $\phi_{f}$. It shows $m i n R_{0}$ is always attained when girls are vaccinated firstly. (b)-(f) Prevalences in the total population with different vaccine distributions for $v=50000$, $v=100000, v=150000, v=250000, v=400000$. The other parameters are from Table 1 . The initial conditions for (b)-(f) are the pre-vaccination endemic equilibria. 
(a)

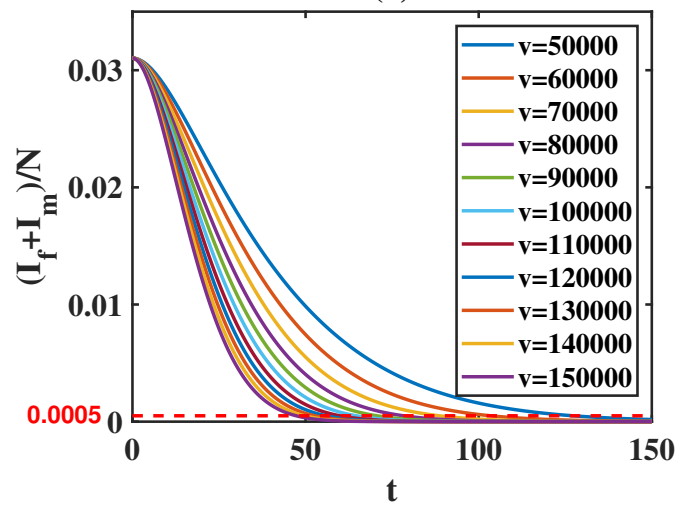

(b)

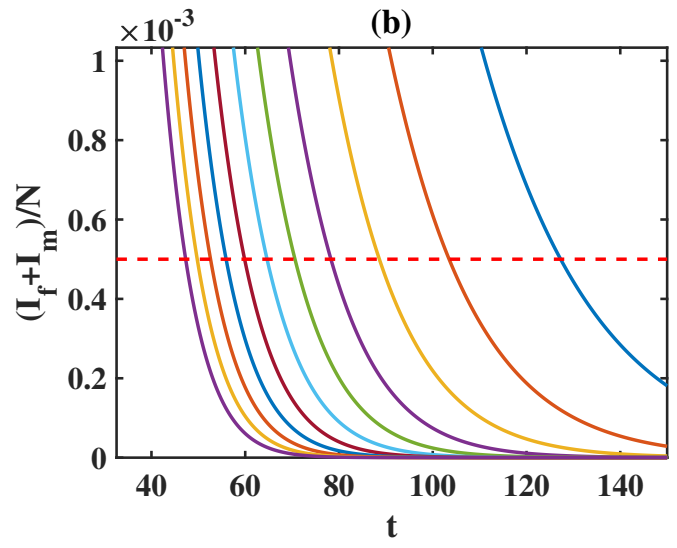

Figure 6: (a) Prevalences in the total population for different vaccine amount $v$ assuming vaccinating girls firstly. All vaccines are given to females. The vaccine coverage can be found in Table 4 . The other parameters are from Table 1. The initial conditions are the pre-vaccination endemic equilibria. (b) Zoomed figure of the lower part of panel (a).

(a)

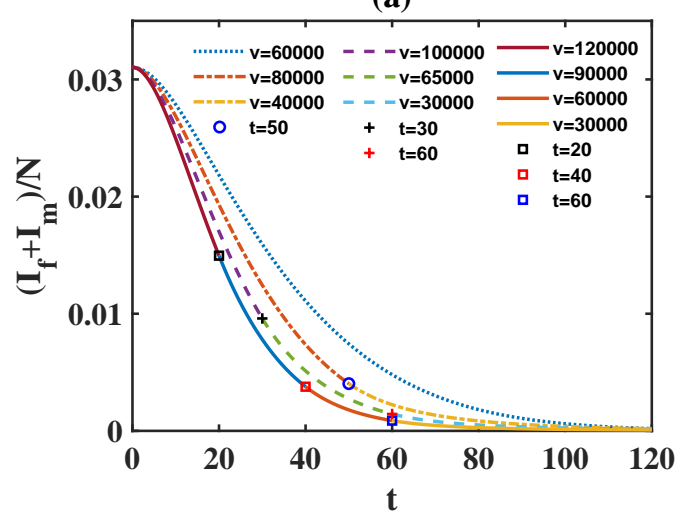

(b)

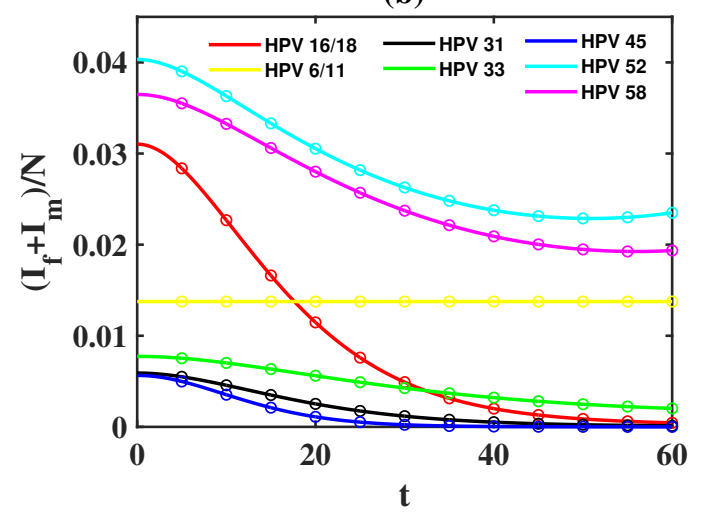

Figure 7: (a) Prevalences in the total population with variable vaccination strategies assuming vaccinating girls firstly. (b) Prevalences in the total population for different HPV types if we allocate vaccine amount $v=175000$ initially and reduce by 15000 every 5 years assuming vaccinating girls firstly and using bivalent vaccines. According to ref. $[53,66]$, we let the degree of protection $\tau$ for HPV 6/11, 31, 33, 45, 52 and 58 be $0,0.5,0.257,0.91,0.372$ and 0.309 , respectively. In the two panels, all vaccines are given to females. The vaccine coverage can be found in Table 5 and Table 6, respectively. The other parameters are from Table 1. The initial conditions are the pre-vaccination endemic equilibria. 
medRxiv preprint doi: https://doi.org/10.1101/2021.12.19.21268067; this version posted December 21, 2021. The copyright holder for this preprint (which was not certified by peer review) is the author/funder, who has granted medRxiv a license to display the preprint in perpetuity.

It is made available under a CC-BY-ND 4.0 International license .

(a)

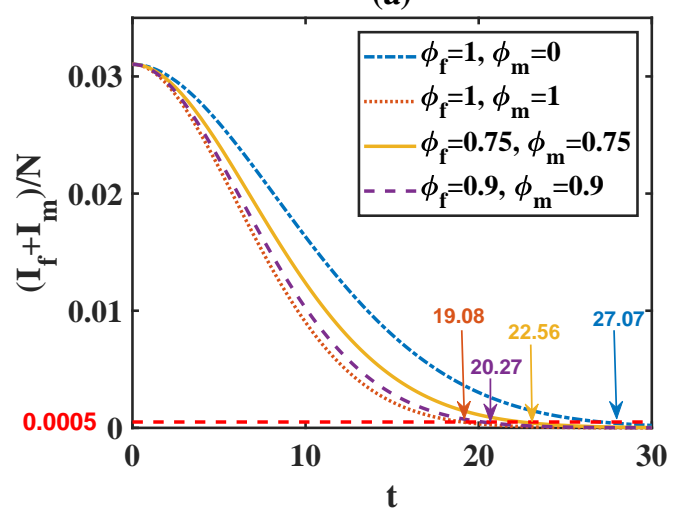

(b)

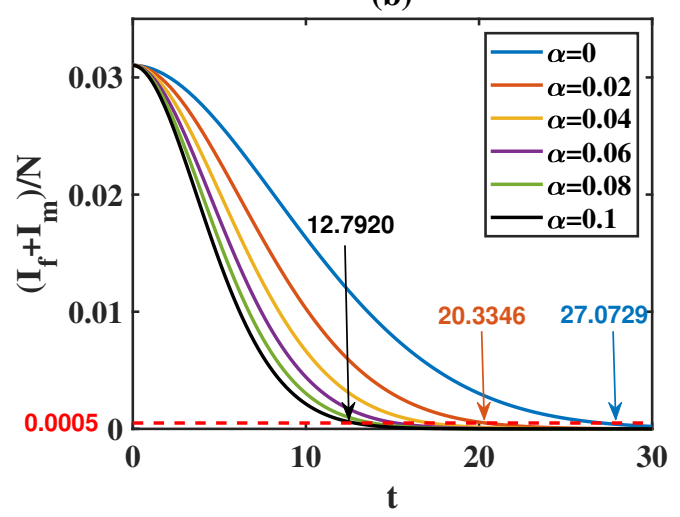

Figure 8: (a) Prevalences in the total population for different vaccination strategies. (b) Prevalences in the total population for different catch-up vaccination proportions with all girls vaccinated by age $15\left(\phi_{f}=1, \phi_{m}=0\right)$. The other parameters are from Table 1 and the initial conditions are the pre-vaccination endemic equilibria. 